University of Tennessee Health Science Center

UTHSC Digital Commons

\title{
Determinants of Physical Activity Behavior and Self-efficacy for Exercise among African American Women
}

Bridget K. Robinson

University of Tennessee Health Science Center

Follow this and additional works at: https://dc.uthsc.edu/dissertations

Part of the Nursing Commons, and the Women's Health Commons

\section{Recommended Citation}

Robinson, Bridget K. , "Determinants of Physical Activity Behavior and Self-efficacy for Exercise among African American Women" (2009). Theses and Dissertations (ETD). Paper 230. http://dx.doi.org/

10.21007/etd.cghs.2009.0263.

This Dissertation is brought to you for free and open access by the College of Graduate Health Sciences at UTHSC Digital Commons. It has been accepted for inclusion in Theses and Dissertations (ETD) by an authorized administrator of UTHSC Digital Commons. For more information, please contact jwelch30@uthsc.edu. 


\title{
Determinants of Physical Activity Behavior and Self-efficacy for Exercise among African American Women
}

\begin{abstract}
Background: Inadequate physical activity behavior persists among African American women despite the widely documented benefits of physical activity. Literature notes a positive correlation between selfefficacy for exercise and physical activity behavior. In addition to socioeconomic factors, culturally influenced factors mediate self-efficacy for task such as physical activity. Past studies indicate differing perceptions related to religiosity and body image among African American populations. Additionally, reports indicate Southern and Midwestern states have the highest prevalence of obesity. These states are located in a geographical area known as the Bible belt. Moreover, $73.5 \%$ of the African American population in the U. S. resides in Bible belt states. Obesity is more prevalent among African American women when compared to other populations. The purposes of this study were to examine the relationships among self-efficacy for exercise, religiosity, body image perception, body mass index, socioeconomic factors (i.e., level of education, monthly income, church attendance, residency [i.e., rural or urban], age, parental responsibility [i.e., caring for children under age 16], number of children, occupation and marital status), and physical activity behavior, to examine differences in the level of the aforementioned variables among that attend church frequently to those that attend church infrequently, and lastly to explicate the predictors of self-efficacy for exercise and physical activity behavior among this sample of African American women.

Methods: This cross-sectional, descriptive, comparative study was used to explore physical activity behavior among African American women. Participants were recruited from a large African Methodist Episcopal church and through word of mouth by women that had completed the study in a Southern city. Participants' self-efficacy for exercise, religiosity, body image perceptions, socioeconomic factors and physical activity behavior were assessed with the following paper and pencil self-report instruments respectively: Physical Exercise Self-efficacy Scale, A Standardized Demographic Data Collection Form, God Locus of Health Control, Appearance Schema Inventory - Revised, and the Stanford Brief Activity Survey. Height and weight information was used to calculate body mass index for each participant. Participants were categorized into groups based on church attendance and physical activity behavior. Participants reported the number of Sunday's they attended church per month on the demographic data collection form and were categorized into the following groups: frequent church attendance (at least 3 times out of the month) and infrequent church attendance (less than 3 times out of the month). The Stanford Brief Activity Survey was used to categorize participants into the following groups inactive (scores ranged from 1-2) and active (scores ranged from 3-5). Descriptive statistics were used to summarize sample characteristics, bivariate correlation analysis was used to examine variable relationships, independent sample t-test and chi-square test were used to examine group differences, and multiple regression analysis was used to determine the predictors of self-efficacy for exercise and physical activity behavior in this sample of African American women.
\end{abstract}

Results: The final sample included 100 African American women. The mean monthly income was $\$ 2,242$. On average participants attended church frequently, had completed high school or attended college, were not married, and had children. Most of the sample was obese (67\%), employed (75\%), resided within the city limits (74\%), and had at least one child under the age of 16 (56\%). Additionally, 61 participants (61\%) reported having occupations that required activity that approximated light intensity physical activity. Many significant relationships were noted among the study variables. Significant relationships were noted between self-efficacy for exercise and physical activity behavior, parental responsibility, body mass index and church attendance. Physical activity behavior was significantly associated with education and type of occupation. Religiosity was significantly associated with education. Body mass index was associated 
with body image perception and education. Groups reported similar levels of religiosity and self-efficacy for exercise. However, women that attended church frequently tended to be younger than those who attended infrequently. Women residing in rural areas reported slightly higher scores on the GLHC indicating extrinsic religiosity. Self-efficacy for exercise predicted physical activity behavior in this sample of African American women. Additionally, the presence of minor children, marital status, and body mass index predicted self-efficacy for exercise.

Conclusion: The findings of this study illuminated significant positive associations between self-efficacy for exercise and physical activity behavior in African American women. Based on the findings of this study, future interventions designed to over come modifiable perceived barriers to physical activity such as having minor children could enhance self-efficacy for exercise and physical activity behavior among African American women. Moreover, further exploration of the influence that being married or partner has on self-efficacy for exercise would be helpful in understanding physical activity behavior among women among this group. Information related to the predictors of self-efficacy for exercise and physical activity behavior will assist researchers plan more tailored culturally relevant health promotion interventions for this population.

\section{Document Type}

Dissertation

\section{Degree Name}

Doctor of Philosophy (PhD)

\section{Program}

Nursing

\section{Research Advisor}

Mona N. Wicks, Ph.D.

\section{Keywords}

African American, Body Image, Physical Activity, Religiosity, Self-efficacy, Women

\section{Subject Categories}

Medicine and Health Sciences | Nursing | Women's Health 


\title{
DETERMINANTS OF PHYSICAL ACTIVITY BEHAVIOR AND SELF- EFFICACY FOR EXERCISE AMONG AFRICAN AMERICAN WOMEN
}

\author{
A Dissertation \\ Presented for \\ The Graduate Studies Council \\ The University of Tennessee \\ Health Science Center
}

\author{
In Partial Fulfillment \\ Of the Requirements for the Degree \\ Doctor of Philosophy \\ From The University of Tennessee
}

By

Bridget K. Robinson

May 2009 
Copyright $@ 2009$ by Bridget K. Robinson

All rights reserved 


\section{DEDICATION}

This dissertation is dedicated to my

Supportive husband Donald, lovely daughters Isis, Tabitha \& Cyan,

Wonderful mother-in-law Barbara

Who loved, nurtured and persevered with me on this memorable journey; and to the Memory of my dear mamma Mrs. Mary A. James who instilled great courage and perseverance in me. 


\section{ACKNOWLEDGEMENTS}

I would like to thank my dissertation chair, Dr. Mona N. Wicks, for helping me accomplish this professional milestone. Dr. Wicks has not only guided my development as a novice researcher, but also mentored and nurtured me, both personally and professionally during my tenure at the University of Tennessee; I am eternally grateful to her for that. Additionally, I would like to thank my entire committee: Dr. Patricia Cowan, Dr. J. Carolyn Graff, Dr. Sharon Husch, and Dr. Denise D. McAdory for sharing their varied expertise with me to facilitate my development as a researcher and to guide this study. I would also like to express special recognition to Dr. Kenneth Robinson, pastor and former Director of Health for the state of Tennessee, who served as a special advisory member of the committee. Pastor Robinson was instrumental throughout every phase of this research study and the insight that he provided regarding the impact of culture on the overall health and well-being of African Americans' will continue to inspire and direct my future research. Finally, I would like to thank my sisters: Valerie and Nicole, my friends, and my colleagues for their continued support and encouragement during this time.

This study was funded by the University of South Alabama College of Nursing Dean's Grant. 


\begin{abstract}
Background: Inadequate physical activity behavior persists among African American women despite the widely documented benefits of physical activity. Literature notes a positive correlation between self-efficacy for exercise and physical activity behavior. In addition to socioeconomic factors, culturally influenced factors mediate self-efficacy for task such as physical activity. Past studies indicate differing perceptions related to religiosity and body image among African American populations. Additionally, reports indicate Southern and Midwestern states have the highest prevalence of obesity. These states are located in a geographical area known as the Bible belt. Moreover, $73.5 \%$ of the African American population in the U. S. resides in Bible belt states. Obesity is more prevalent among African American women when compared to other populations. The purposes of this study were to examine the relationships among self-efficacy for exercise, religiosity, body image perception, body mass index, socioeconomic factors (i.e., level of education, monthly income, church attendance, residency [i.e., rural or urban], age, parental responsibility [i.e., caring for children under age 16], number of children, occupation and marital status), and physical activity behavior, to examine differences in the level of the aforementioned variables among that attend church frequently to those that attend church infrequently, and lastly to explicate the predictors of self-efficacy for exercise and physical activity behavior among this sample of African American women.
\end{abstract}

Methods: This cross-sectional, descriptive, comparative study was used to explore physical activity behavior among African American women. Participants were recruited from a large African Methodist Episcopal church and through word of mouth by women that had completed the study in a Southern city. Participants' self-efficacy for exercise, religiosity, body image perceptions, socioeconomic factors and physical activity behavior were assessed with the following paper and pencil self-report instruments respectively: Physical Exercise Self-efficacy Scale, A Standardized Demographic Data Collection Form, God Locus of Health Control, Appearance Schema Inventory - Revised, and the Stanford Brief Activity Survey. Height and weight information was used to calculate body mass index for each participant. Participants were categorized into groups based on church attendance and physical activity behavior. Participants reported the number of Sunday's they attended church per month on the demographic data collection form and were categorized into the following groups: frequent church attendance (at least 3 times out of the month) and infrequent church attendance (less than 3 times out of the month). The Stanford Brief Activity Survey was used to categorize participants into the following groups inactive (scores ranged from 1-2) and active (scores ranged from 3-5). Descriptive statistics were used to summarize sample characteristics, bivariate correlation analysis was used to examine variable relationships, independent sample t-test and chi-square test were used to examine group differences, and multiple regression analysis was used to determine the predictors of self-efficacy for exercise and physical activity behavior in this sample of African American women.

Results: The final sample included 100 African American women. The mean monthly income was $\$ 2,242$. On average participants attended church frequently, had completed 
high school or attended college, were not married, and had children. Most of the sample was obese (67\%), employed (75\%), resided within the city limits (74\%), and had at least one child under the age of $16(56 \%)$. Additionally, 61 participants $(61 \%)$ reported having occupations that required activity that approximated light intensity physical activity. Many significant relationships were noted among the study variables. Significant relationships were noted between self-efficacy for exercise and physical activity behavior, parental responsibility, body mass index and church attendance. Physical activity behavior was significantly associated with education and type of occupation. Religiosity was significantly associated with education. Body mass index was associated with body image perception and education. Groups reported similar levels of religiosity and self-efficacy for exercise. However, women that attended church frequently tended to be younger than those who attended infrequently. Women residing in rural areas reported slightly higher scores on the GLHC indicating extrinsic religiosity. Self-efficacy for exercise predicted physical activity behavior in this sample of African American women. Additionally, the presence of minor children, marital status, and body mass index predicted self-efficacy for exercise.

Conclusion: The findings of this study illuminated significant positive associations between self-efficacy for exercise and physical activity behavior in African American women. Based on the findings of this study, future interventions designed to over come modifiable perceived barriers to physical activity such as having minor children could enhance self-efficacy for exercise and physical activity behavior among African American women. Moreover, further exploration of the influence that being married or partner has on self-efficacy for exercise would be helpful in understanding physical activity behavior among women among this group. Information related to the predictors of self-efficacy for exercise and physical activity behavior will assist researchers plan more tailored culturally relevant health promotion interventions for this population. 


\section{TABLE OF CONTENTS}

CHAPTER 1. INTRODUCTION .................................................................................1

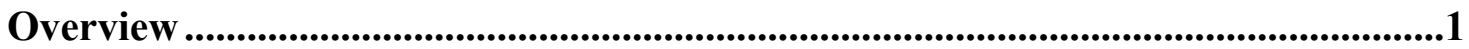

Purpose of the Study ................................................................................................................4

Specific Aim 1 ........................................................................................6

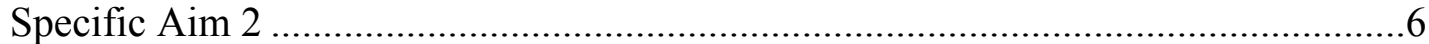

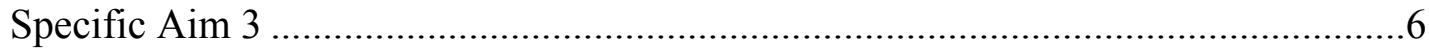

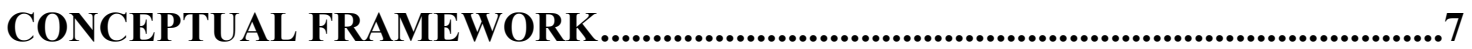

Expanded Conceptual Framework .................................................................................7

Antecedent Conditions/Contributory Factors …………............................................

Consequences/Outcomes .................................................................................

Definitions of Major Concepts .........................................................................................11

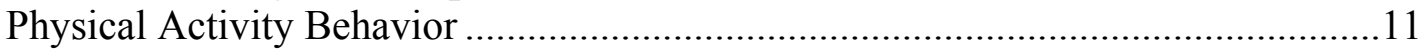

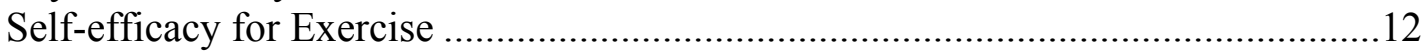

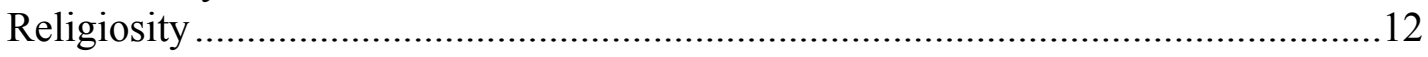

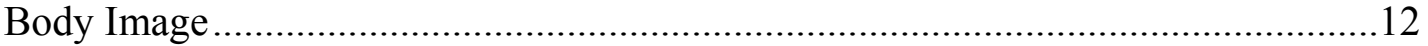

Residency ............................................................................................13

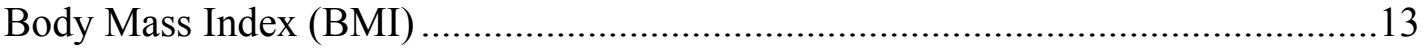

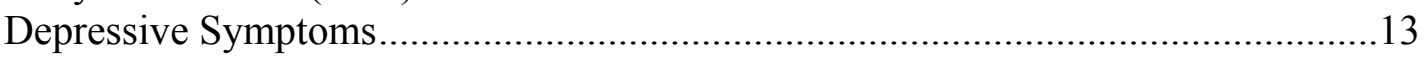

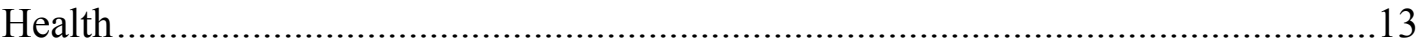

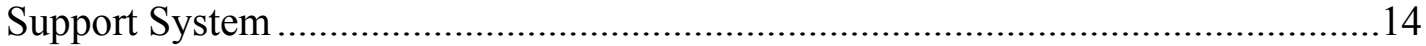

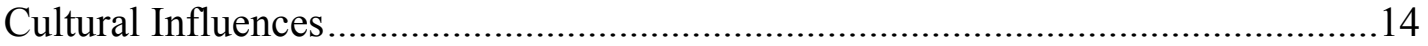

Significance of Study..................................................................................................14

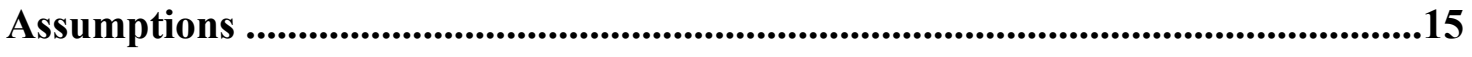

Limitations......................................................................................................................16

CHAPTER 2. REVIEW OF LITERATURE ............................................................18

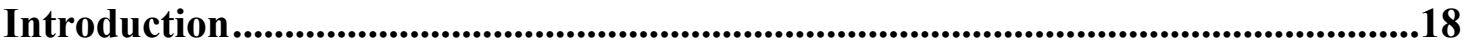

Physical Activity ................................................................................................................19

Self-efficacy for Exercise ……………………………..................................19

Socioeconomic Factors and Self-efficacy for Exercise ........................................20

Body Image Perception and African American Culture ..........................................21

Body Image Perception and African American Women ..........................................22

Religiosity and African American Culture …………...........................................23

Religiosity and African American Women..........................................................24

Physical Activity, Religiosity, and African American Women.................................25

Summary of the Review of Literature ..............................................................................26

CHAPTER 3. METHODOLOGY ...............................................................................28 
Research Design ....................................................................................................................28

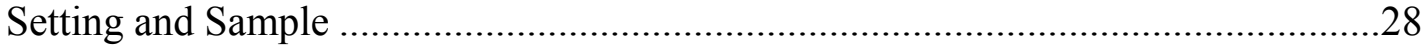

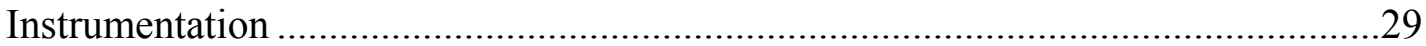

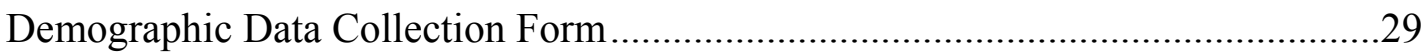

Physical Exercise Self-efficacy Scale ....................................................................30

God Locus of Health Control...............................................................................30

Stanford Brief Activity Survey …………………….............................................31

Appearance Schema Inventory - Revised ..............................................................32

Procedure .......................................................................................................................33

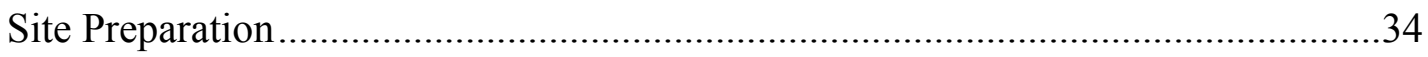

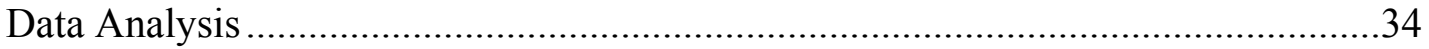

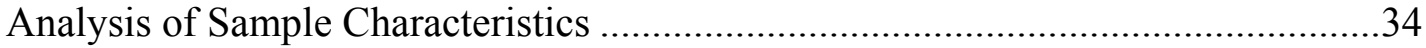

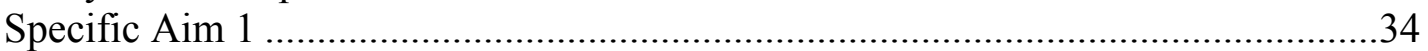

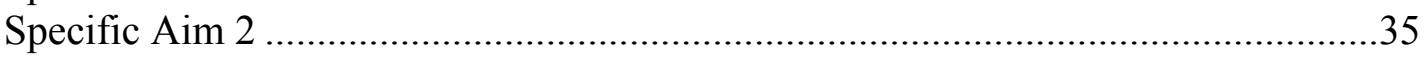

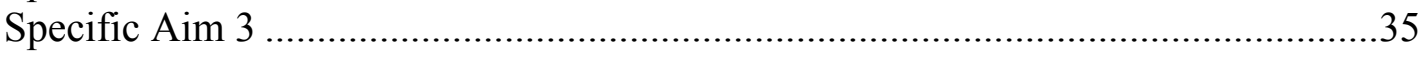

Consideration of Human Subjects.....................................................................................36

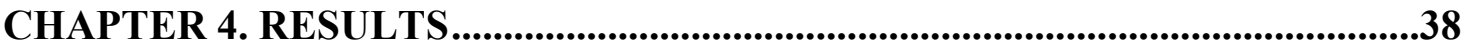

Sample Characteristics................................................................................................38

Research Aims, Related Questions, and Findings.........................................................42

Specific Aim 1 ......................................................................................43

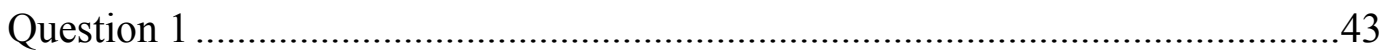

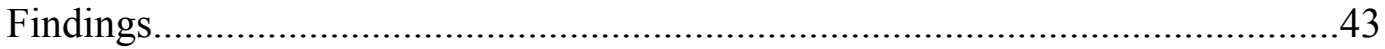

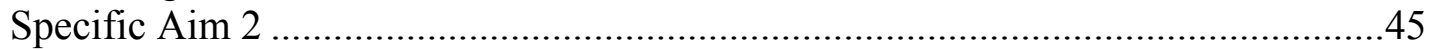

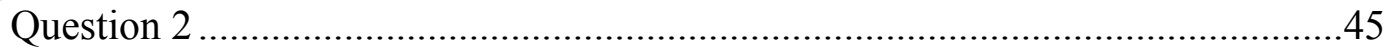

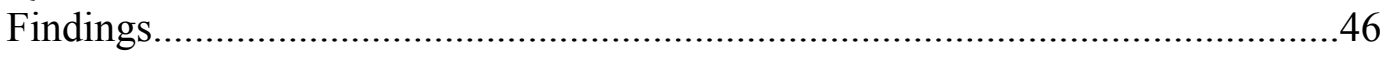

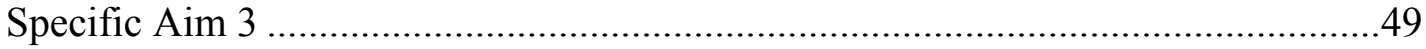

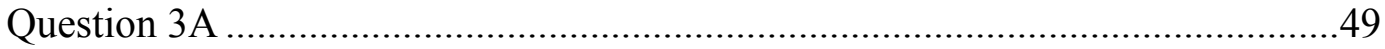

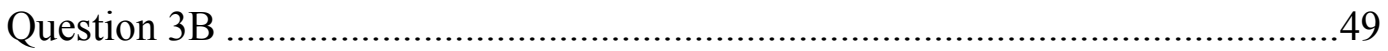

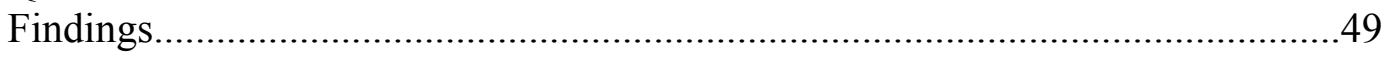

CHAPTER 5. DISCUSSION AND IMPLICATIONS............................................53

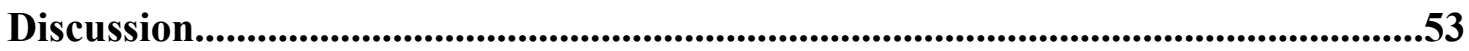

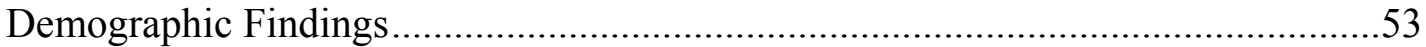

Relationships among Self-efficacy for Exercise, Religiosity, Body Image

Perception, Body Mass Index, Socioeconomic Factors and Physical Activity

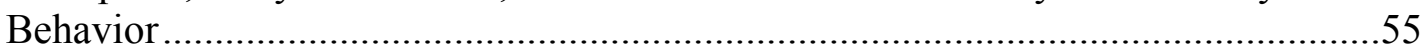

Group Differences: Frequent Church Attendance and Infrequent Church

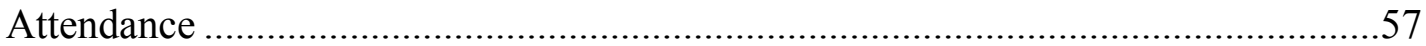

Predictors of Self-efficacy for Exercise and Physical Activity Behavior...................57

Limitations and Strengths.................................................................................................58

Implication: Theory, Research, and Practice...................................................................60

Summary and Recommendations for Further Research ............................................61 
LIST OF REFERENCES ..............................................................................63

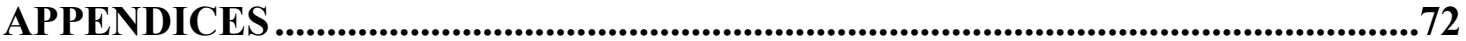

Appendix A. Written Permission/Proof of Purchase for Study Instruments........73

Appendix B. Standardized Demographic Data Form ..............................................75

Appendix C. Physical Exercise Self-Efficacy Scale............................................76

Appendix D. God Locus of Health Control Scale .......................................................77

Appendix E. Stanford Brief Activity Survey ............................................................78

Appendix F. Appearance Schema Inventory-Revised ........................................81

Appendix G. Institutional Review Board Approval Letter.....................................83

Appendix H. Approval Letter from the Pastor of St. John AME Church ............84

VITA 


\section{LIST OF TABLES}

Table 1. Demographic Characteristics of This Sample of African American

Women

Table 2. Summarized Data for Physical Activity, Self-efficacy for Exercise,

Religiosity, and Body Image Perception

Table 3. Summarized Data for Bivariate Correlation Analyses

Table 4. Chi-square Cross Tabulation of Demographic and Categorized Outcome Group Differences between African American Women That Attend Church Frequently and Those That Attend Infrequently

Table 5. Dummy Codes of Categorical String Variables ........................................ 50

Table 6. Summary of Multiple Regression Analysis for Self-efficacy for Exercise $(N=100)$.

Table 7. Summary of Multiple Regression Analysis for Physical Activity

Behavior $(N=100)$. 52 


\section{CHAPTER 1. INTRODUCTION}

Inadequate physical activity behavior persists among African American women despite the widely documented benefits of physical activity (Centers for Disease Control and Prevention [CDC], 2002; Flynn \& Fitzgibbon, 1998; Taylor-Piliae et al., 2006; U.S. Department of Health and Human Services [USDHHS], 1996). Literature notes a positive correlation between self-efficacy for exercise and physical activity behavior. In addition to socioeconomic factors, culturally influenced factors mediate self-efficacy for tasks such as physical activity. Additionally, differing perceptions related to religiosity and body image have been noted among African American populations. According to the Trust for America's Health (2008), Southern and Midwestern states have the highest prevalence of obesity. These states are located in a geographical area known as the Bible belt. Moreover, $73.5 \%$ of the African American population in this nation reside in the Bible belt states (Trust for America's Health, 2008). Obesity characterized by higher body mass index (BMI) is more prevalent among African American women when compared to other populations. The current study examines the dynamic relationships among self-efficacy for exercise, religiosity, body image perception, BMI, socioeconomic factors, and physical activity behavior among African American women.

\section{Overview}

Physical activity, a strategy often prescribed to eliminate obesity, is associated with improved mental and physical health outcomes (Ard \& Rosati, 2000; Ball et al., 2007; Ester, Mireille, Jos, \& Twisk, 2006; Flynn \& Fitzgibbon, 1998; Franzoni \& Ghiadoni, 2005; Jones, 1992; Probst, Moore, Glover, \& Samuels, 2004; Staffileno, Coke, \& Hollenberg, 2007). Approximately $60 \%$ of Americans are physically inactive despite the documented health benefits of this lifestyle behavior; the estimated rate of inactivity is higher among minority populations especially among African American women (Staffileno et al., 2007). Though the best weight loss results occur when dietary strategies are combined with exercise, routine physical activity alone produces significant health benefits including improved glucose tolerance, decreased systolic blood pressure, decreased cardiac work, and improved mood (Craft, Freund, Cupepper, \& Perna, 2007; Probst et al., 2004; Staffileno et al., 2007). Thus, increased physical activity behavior among African Americans could produce many health and economic benefits that include decreased prevalence of obesity, decreased prevalence of obesity-related health disparities, and significant reduction in associated public health costs. Self-efficacy is a central concept in Bandura's (1997) social-cognitive theory and has been shown to be a more effective measure of the likelihood to adhere to behavior change, than expectancy outcomes (Schwarzer \& Renner, 2000). Perceived self-efficacy reflects an individual's confidence in their ability to perform a specific task such as physical activity (Schwarzer \& Renner, 2000; Staffileno et al., 2007). In the current study self-efficacy for exercise is the measure of participants' confidence in their ability to engage in physical activity. 
The degree of self-efficacy for exercise that is present is a critical factor that influences health practices such as physical activity behavior (Wilbur, Miller, Chandler, \& McDevitt, 2003; Wolfe, 2000). Consequently, a change in physical activity behavior depends on an individual's self-efficacy for exercise. Dynamic interactions among many factors, referred to as correlates in this study, mediate self-efficacy for exercise.

Socioeconomic factors such as income, educational attainment, and type of occupation could impact self-efficacy for exercise. African American women are often described in the literature as single parents. The affective and financial implications of heading a single parent household could impact an individual's confidence in their exercise intentions as well as impede actual physical activity engagement. Moreover, African American women cite culture as the source for a greater perceived family responsibility that serves as a barrier to engaging in physical activity. Thinking patterns, value systems, and moral/emotional states are internal processes integral to self-efficacy for exercise and influenced by culture through interpersonal relationships (Bandura, 1997; Dornelas, Stepnowski, Fischer, \& Thompson, 2007). The external process of selection, a component of self-efficacy for exercise, is highly individualized and affected by choices such as physical activity behavior (Bandura, 1997; Dornelas et al., 2007). One way that culture influences perceptions or schemas is through social or vicarious learning. Environments that promote health consciousness and emphasize the positive health benefits associated with physical activity while facilitating engagement in physical activity would likely influence self-efficacy for exercise. African American women participate in organized religious activities, such as attending church at a higher rate when compared to other populations (K. S. Johnson, Elbert-Avila, \& Tulsky, 2005). Health promotion strategies that incorporate physical activity into established or organized religious activities could positively affect physical activity behavior among African American women. Thus promotion of physical activity, as well as engagement in physical activity within the African American community could offer opportunities for vicarious learning and thus would influence personal schemas related to physical activity behavior in this group. Additionally, thinking patterns related to personal versus divine control over health status determine an individual's degree of religiosity and could affect self-efficacy for exercise.

The literature includes many studies that document the significant association between religiosity and physical health; however there is limited empirical evidence delineating the relationship between religiosity and psychological outcomes such as selfefficacy for exercise (Milevsky \& Levitt, 2004). It is essential to consider the dimensions of religiosity in research studies that explore this construct. Intrinsic and extrinsic religiosity exerts different effects on the psyche (Milevsky \& Levitt, 2004). Religion may exert an external effect on control related cognition (Wallston et al., 1999). Compared with Caucasian participants, African Americans in one study had a higher degree of religiosity and a religious locus of control that placed responsibility for their health and illness on God as a means of coping (K.S. Johnson et al., 2005). Referred to as God locus of health control (GLHC), this extrinsic religiosity may affect behavior outcomes, such as engagement in physical activity (Murray, Goggin, \& Malcarne, 2006). African Americans, compared to other populations report greater participation in organizational and non-organizational religious activities. The level of participation in these activities 
may be associated with a history of perceived oppression and mistreatment (K. S. Johnson et al., 2005). Although several investigators have examined the role of religious beliefs with regard to understanding, coping with, and recovering from illness, few studies have focused on the role of religious beliefs related to health promotion and disease prevention in African American adults (K. S. Johnson et al., 2005). Greater understanding of the multidimensional construct of religiosity and its effects on selfefficacy for exercise could be beneficial to health promotion efforts in African American communities. The African Methodist Episcopal (AME) Church, an organization formed by freed slaves, is a place where members of this population have traditionally shared social and cultural support (Resnicow, Baranowski, Ahluwalia, \& Braithwaite, 1999; Wilcox et al., 2007; Yenek, Becker, Moy, Gittelsohn, \& Koffman, 2001). African American churches offer a unique opportunity within the context of culture for inquiry and introduction of population wide interventions addressing the obesity epidemic (Resnicow et al., 2002; Wilcox et al., 2007; Yenek et al., 2001).

African American populations experience obesity and other health problems at a disproportionate rate, in part because of cultural factors and historical events. Slavery, mistreatment, and conditions associated with poverty that are transmitted between generations could account for common schemas related to religion, physical activity, and body image among members of this cultural group (Holt, Lewellyn, \& Rathweg, 2005). African American women may hold differing views related to body image and weight when compared to other ethnic groups. Some studies attribute the obesity epidemic to a relative non-preoccupation with weight among African American populations (Wolfe, 2000). Additionally, the aforementioned attitude is often referred to as a cultural norm and should be considered when examining obesity among African Americans.

Obesity is a growing problem throughout the world with noted differences in the rate of increase based on not only ethnicity but also residency location. Research conducted by Plantinga and Bernell (2004) suggests a more rapid rise in the rate of obesity among individuals that reside in rural areas without regard to ethnicity. Moreover, Probst et al. (2004) reported that $75 \%$ of African Americans resided in the rural Southeastern states, a portion of which is known as the Bible belt. However, cumulative rural statistics reflect an 84\% Caucasian, 8\% African American, and 5\% other ethnic group population distribution (Powell, Slater, Chaloupka, \& Harper, 2006). These conflicting statistics could obscure the obesity epidemic among rural dwelling African Americans.

Exploration of the complex association between access to settings that facilitate participation in physical activity, actual engagement in physical activity, and obesity in rural populations could provide valuable information to guide the development of future health promotion strategies in this area (Plantinga \& Bernell, 2004). There is a growing body of empirical data that documents community level correlates of obesity, and that indicate the need for focused environmental and policy intervention strategies to reduce population-level obesity prevalence (Plantinga \& Bernell, 2004). Rural and urban differences in access to community walking tracks, gyms, and parks could account for the more rapid rise in obesity in the rural community. Additionally, factors such as fear, poor 
or no sidewalks, and inclement weather are common barriers to participating in routine physical activity (Clark \& Nothwehr, 1999; Powell et al., 2006).

The association between socioeconomic status, self-efficacy for exercise and physical activity has been noted. According to Probst et al. (2004), the incidence of poverty for rural residents was $28 \%$ higher than for urban residents. Additionally, $39.5 \%$ of African American adults living in rural areas did not have a high school diploma. Of particular concern is the fact that in the south, educated African American residents often migrate to urban areas while less educated African Americans often migrate to rural areas (Probst et al., 2004). The prevalence of lower socioeconomic status and a lower level of education could contribute to the growing physical inactivity among rural dwelling African American residents. The current study examined physical activity behavior among African American women that resided in rural areas.

\section{Purpose of the Study}

Despite literature that points out the benefits of being physically activity there has been little progress towards sustained increases in African American women (CDC, 2002; Flynn \& Fitzgibbon, 1998; USDHHS, 1996; Wilbur et al., 2003; Wolfe, 2000). African American women continue to experience obesity and obesity-related health complications at a disproportionate rate. Members of this group have noted access to resources and environmental and social support as facilitators to being physically active. Additionally, African American women cite barriers such as family/parental responsibility, financial burden, and weather. While several researchers have used the social-cognitive theory framework to plan and implement interventions few have identified specific modifiable barriers relevant to African American women. These studies have primarily used the faith-based and faith-placed strategy to address cultural sensitivity in their study design. However, simply implementing interventions through the African American church setting may not fully mediate the effects of culture in this population (Resnicow et al., 1999). Although church-based interventions aid in the recruitment aspect of the studies, retention which is essential for sustained impact remains an issue. High attrition rates ( $>40 \%$ at follow-up) in past studies incorporating church/faith-based interventions evidence retention issues with this approach. Identifying and focusing on modifiable barriers such as affordable or free access to venues for physical activity engagement and child care accommodations could minimize issues related to attrition.

Several studies have addressed health promotion related to diet and physical activity through interventions in the African American church with mixed results. Wilcox et al. (2007) conducted the Healthy-AME faith-based intervention study and used trained volunteers to implement their study. These investigators found that health information awareness, along with fruit and vegetable consumption increased over the two-year period of the study but physical activity did not. The WORD (Wholeness, Oneness, Righteousness, Deliverance) study was an eight week behaviorally focused weight loss study that focused on diet and exercise (Kim et al., 2006). Although participants reported 
relatively small increases in recreational physical activity compared to other intervention studies, the authors cited the eight week length of the intervention (pilot study) as a likely explanation for the difference. Additionally, the investigators only reported recreational physical activity in the WORD study. Researchers should consider intensity and duration of physical activity behavior when establishing cardiovascular benefit. The goal of the Project Joy study conducted by Yenek et al. (2001) was to reduce cardiovascular risk factors in African American women. Although researchers achieved most of their program outcomes, there was no significant increase in physical activity. The authors noted a substantial decrease in attendance in their volunteer led intervention group in comparison to the staff led group and found that participants did not feel comfortable being advised by their peers (Yenek et al., 2001). This attitude could explain, in part, the failure to meet the physical activity outcome in the Healthy-AME and Project Joy studies. The information obtained in this current descriptive study that was designed to gain insight into the effect of culturally influenced perceptions, as explained through the social-cognitive theory, could be beneficial in planning and implementing more effective intervention studies to address physical activity behavior issues among African Americans. The proposed study sought to accomplish this vital initial step.

Among the predictors of physical activity are the factors that affect self-efficacy for exercise including expected benefits of physical engagement and social support (Green et al., 2006). Social and cultural barriers such as lower social economic status, lower education level, lack of motivation, and altered body image perception are possible explanations for the continued physical inactivity and the obesity disparity in African American populations (Green et al., 2006). Cultural influences and social structures play a collective role in the African American psyche (Ball et al., 2007; Jones, 1992). Some investigators have partially addressed one aspect of the effects of culture and social structure on self-efficacy for exercise by placing their interventions in the church; however careful examination of religiosity and self-efficacy for exercise could further illuminate physical activity behavior among African American women. An individual's degree of religiosity could affect the receipt of benefits from weight loss programs in African American populations (Kim et al., 2006) and should be explored. Churches in African American communities provide emotional and social support to parishioners, a type of support that is integral to sustained self-efficacy for exercise and physical activity engagement. Exploration of the relationship between body image perception, selfefficacy for exercise, religiosity, and physical activity among African American women in a church environment could provide useful insight into risky health behaviors such as physical inactivity.

Interventions planned and implemented following the Social-Cognitive theoretical framework may decrease the rate of obesity in African American populations better than interventions based on other theories (Ball et al., 2007; Wilbur et al., 2003). Wilcox et al. (2007) used the social ecological and transtheoretic models as conceptual frameworks. They combined these frameworks to capture the effects of social networks on behavior and individual readiness to change. Although theses researchers have addressed two very important factors that affect behavior, they have neglected to recognize the impact that personal factors, such as emotions have on behavior. The use of social-cognitive theory, 
which holistically considers personal belief systems, personal motivations, emotions, and personal choices, would capture the various effects of culturally influenced perceptions and better explain physical activity behavior among African American women. The current study explored these factors through examination of the following specific aims and related research questions:

\section{Specific Aim 1}

Explore the relationships among self-efficacy for exercise, religiosity, body image perception, body mass index, socioeconomic factors (i.e., level of education, monthly income, church attendance, residency [i.e., rural or urban], age, parental responsibility [i.e., caring for children under age 16], number of children, occupation and marital status), and physical activity behavior among African American women.

Research Question 1: What are the relationships among self-efficacy for exercise, religiosity, body image perception, body mass index, level of education, monthly income, church attendance, residency (i.e., rural or urban), age, parental responsibility (i.e., caring for children under age 16), number of children, occupation, marital status, and physical activity behavior among African American women?

\section{Specific Aim 2}

Compare the level of self-efficacy for exercise, religiosity, body image perception, body mass index, socioeconomic factors (i.e., level of education, monthly income, church attendance, residency [i.e., rural or urban], age, parental responsibility [i.e., caring for children under age 16], number of children, occupation and marital status), and physical activity behavior among African American women that regularly attend church services (at least 3 times out of the month) to those who do not regularly attend church services (less than 3 times out of the month).

Research Question 2: What are the differences in self-efficacy for exercise, religiosity, body image perception, body mass index, socioeconomic factors and physical activity behavior among African American women that regularly attend church services (at least 3 times out of the month) compared to those who do not regularly attend church services (less than 3 times out of the month)?

\section{Specific Aim 3}

Explicate the predictors of self-efficacy for exercise and physical activity behavior among African American women.

Research Question 3A: What are the predictors of self-efficacy for exercise among African American women? 
Research Question 3B: What are the predictors of physical activity behavior among African American women?

\section{CONCEPTUAL FRAMEWORK}

Bandura's social-cognitive theory guided this study as it included key concepts that reflect the study variables. Moreover, this theory influenced instrument selection and the study design. The social-cognitive theory describes a dynamic interaction (Figure 1) between behavior, personal factors, and environmental factors (Green et al., 2006). Perceived self-efficacy is the focus of social-cognitive theory and is defined as one's personal belief in the ability to facilitate change within a specified area of one's life (Bandura, 1997), which was physical activity in the current study. Perceived self-efficacy for exercise is a driving force in efforts towards commitment to routinely engage in physical activity (Figure 2) for an extended period of time (Schwarzer \& Renner, ND). A high self-efficacy for exercise results in commitment to routine physical activity and improved health outcomes, whereas a low self-efficacy for exercise results in inconsistent physical activity and poorer health outcomes (Green et al., 2006).

The current study incorporated key aspects of the social-cognitive theory (Figure 1). This basic model depicts the dynamic interaction among personal experiences, cognitions, motivations, affect, individual choices, and behavior. This study examined pertinent variables thought to affect self-efficacy for exercise and physical activity behavior in African American women under each domain of the social-cognitive theory. An expanded conceptual model (Figure 2) depicts the author's interpretation of the variables examined in this study. The expanded conceptual model depicts the proposed relationships among self-efficacy for exercise, religiosity, body image perception (BI), body mass index (BMI), level of education, monthly income, church attendance, residency, age, children, occupation, marital status, and physical activity behavior. This study only examined those variables marked with an asterisk on the expanded conceptual model (Figure 2). Additionally, the solid lines on the expanded conceptual model represent the relationships explored in this study.

\section{Expanded Conceptual Framework}

\section{Antecedent Conditions/Contributory Factors}

Personal schemas related to exercise, which develop during the formative years, are influenced by mastery of experience, vicarious learning, social persuasion, and emotional affect (Bandura, 1994). There may be limited opportunities for engagement in or observation of others engaging in physical activity in the African American culture, so although physical activity is a task that has most likely been mastered during school years, the lack of personal models and social persuasion within this community has not 


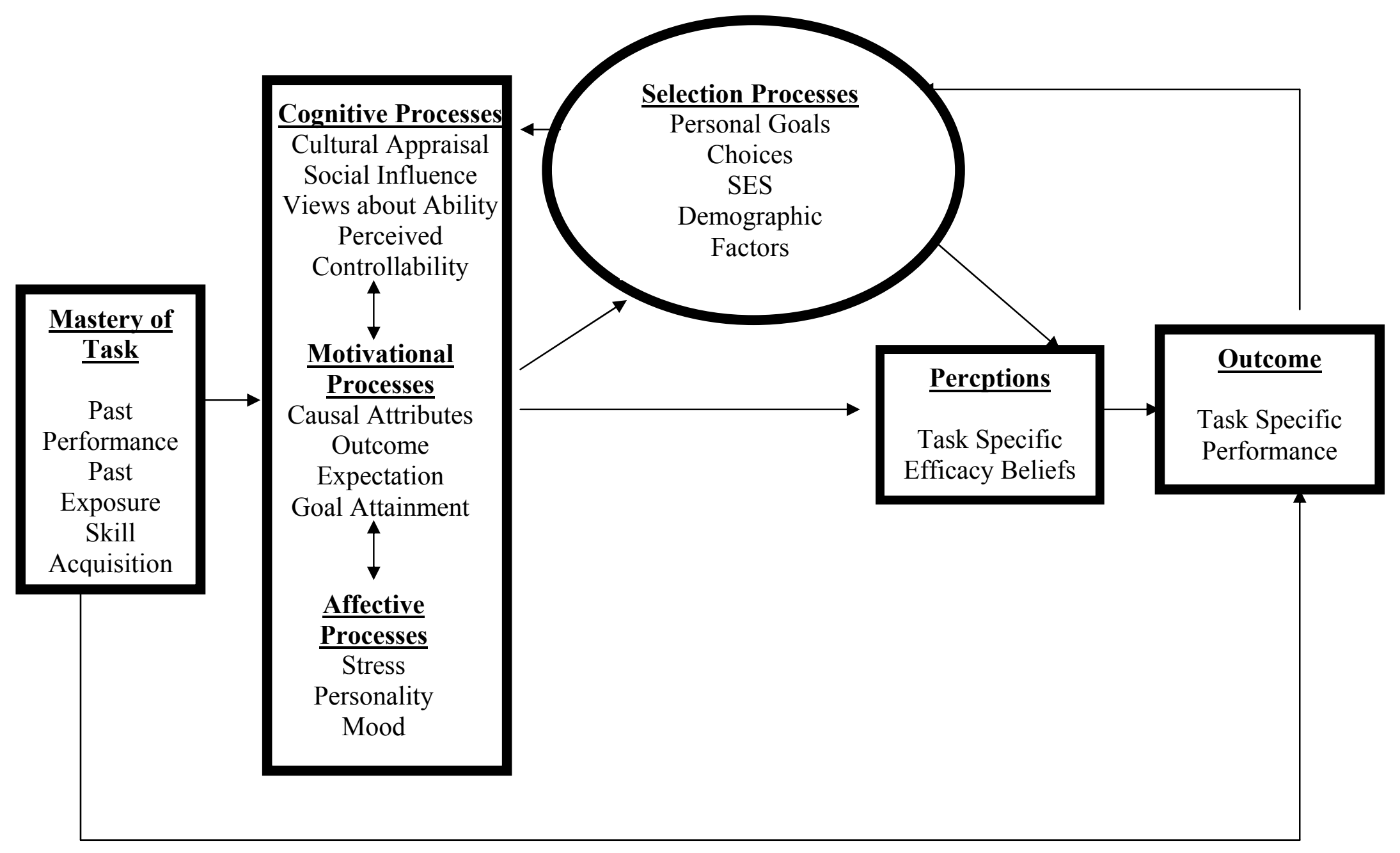

Figure 1. Conceptualized model of the social-cognitive theory. 


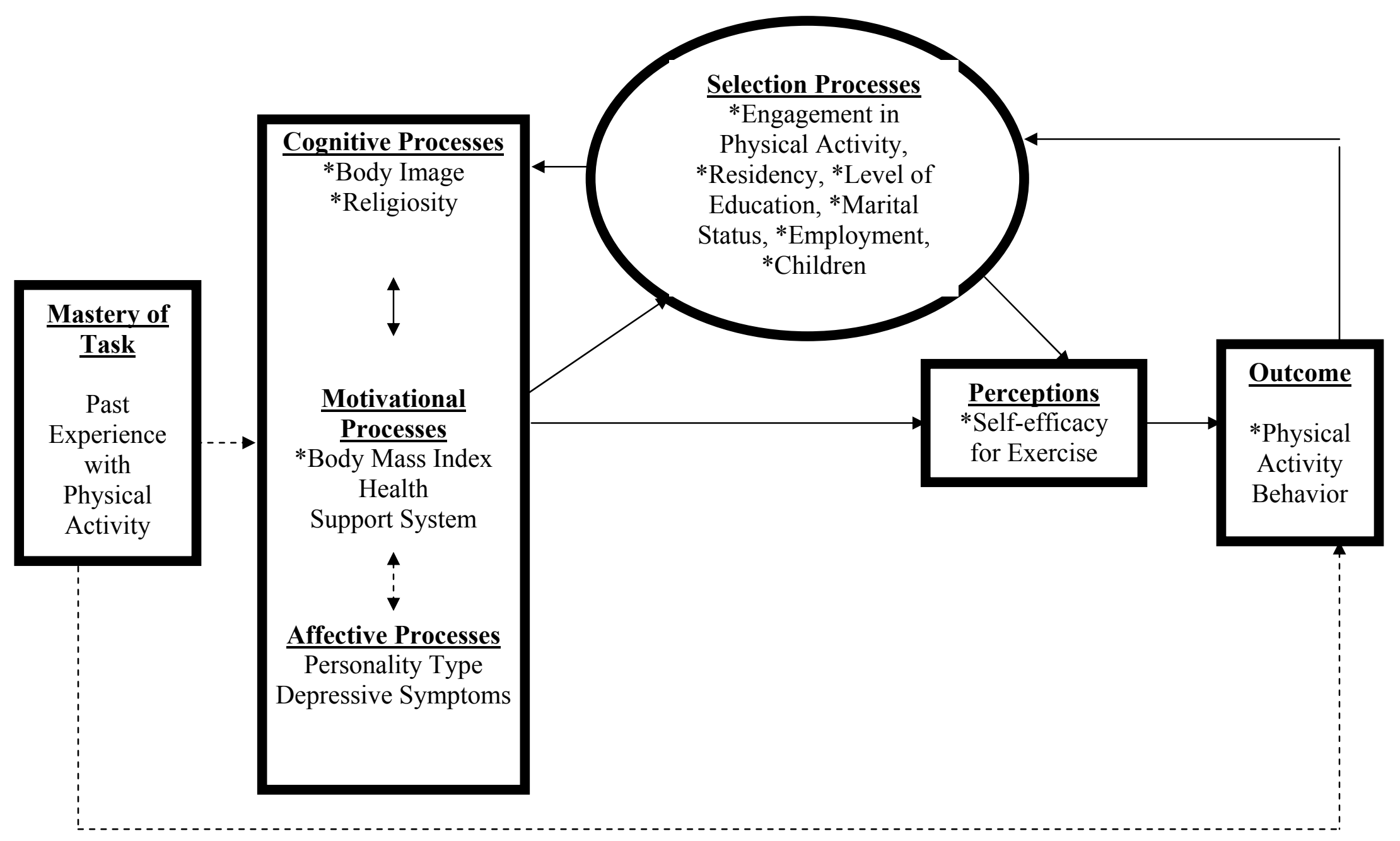

Figure 2. Expanded conceptual model of the social-cognitive theory. 
facilitated in most cases a high self-efficacy for exercise. Many factors such as limited mobility related to higher BMI, low income, and low educational level could result in result in limited exposure to information related to various ways of engaging in physical activity (Clark \& Nothwehr, 1999). The above factors are prevalent in African American populations and could produce or exacerbate a low self-efficacy for exercise. Vicarious learning is a major component of self-efficacy for exercise, thus if an individual is not exposed to environments where physical activity is routinely taking place, there will be decreased levels of self-efficacy for exercise. An individual's choices about the places they live, attend school, obtain jobs, attend church, and socialize will expose them to opportunities for vicarious learning and ultimately affect their level of self-efficacy for exercise. Attending church and residing in a rural community could limit access to a venue for physical activity and affect self-efficacy for exercise (Plantinga \& Bernell, 2004). According to Holt et al. (2005) there may be few opportunities for modeling of health protective behaviors in the African American church.

Individual perception about body image will affect self-efficacy for exercise. Consideration of the source of one's social persuasion should be determined when examining self-efficacy for exercise. In the African American culture, it is acceptable and sometimes expected to have a higher BMI, so achieving the recommended body weight may not serve as adequate motivation to be physically active for members of this community (Wolfe, 2000). Perception of health is associated with religion. According to Milevsky and Levitt (2004) religiosity is significantly and positively correlated with physical health. An intrinsic religiosity, which seems to empower individuals, has been associated with motivating healthy behaviors such as abstinence and smoking cessation, while an extrinsic religiosity, which seems to enable, has been associated with unhealthy behaviors such as not doing self breast exams (Milevsky \& Levitt, 2004). Religiosity produces a control related cognition which has an overarching effect that permeates the concept of self-efficacy for exercise (Holt et al., 2005). Individual sources of social support impact affective mood and self-efficacy for exercise. Family members, friends, and even pastors can serve as either social support or barriers to changing health behaviors. Holt et al. (2005) found that social support was a religion-health mediator in a sample of African American parishioners.

Overall affective processes will influence self-efficacy for exercise. Depression, fear, and anxiety can all impact one's level of physical activity. A major factor that affects routine engagement in physical activity is the identification of practical, convenient, and achievable forms of physical activity (Nies, Vollman, \& Cook, 1999). If obese individuals have fear and anxiety related to working out in a gymnasium full of physically fit individuals, their level of self-efficacy for exercise will be diminished, they may exhibit depressive symptoms, and they will be less likely to engage in physical activity. So, simply encouraging these individuals to go to the gymnasium would not be sufficient, practical, or convenient alone to address physical inactivity without addressing the fear and anxiety that may be associated with going to the gymnasium. Additionally, personality type has been associated with affective mood. For example, "type A" personalities have been linked to anger, depression, and anxiety, thus this personality trait is a contributory factor related to self-efficacy for exercise (Bettencourt, Talley, 
Benjamin, \& Valentine, 2006). The "type A" personality trait directly affects an individual's affective mood, hence making it relevant to the social-cognitive theory.

\section{Consequences/Outcomes}

The commitment to engage in routine physical activity for a sustained period of time is dependant in part upon an individual's perception of the usefulness and personal benefits associated with this activity. An attitude of confidence in the ability to engage in physical activity, positive beliefs about the personal benefit of physical activity, and engagement in physical activity have been associated with routine physical activity (Green et al., 2006). Higher self-efficacy for exercise is associated with engagement in physical activity which leads to decreased obesity-related complications, and an overall increased sense of wellbeing. Low self-efficacy for exercise is associated with apprehension, anxiety, and less motivation to act (Holt et al., 2005). When there are deficits or negative aspects related to contributory factors associated with self-efficacy for exercise, such as lack of concern over body image, decreased engagement in physical activity, and depressive symptoms, a lower self-efficacy for exercise and poorer health outcomes result (Clark \& Nothwehr, 1999; Green et al., 2006). Low self-efficacy for exercise predisposes to obesity and many chronic illnesses such as depression, coronary artery disease, hypertension, and type 2 diabetes mellitus. The effects of self-efficacy for exercise are cyclic in nature (Green et al., 2006). Individual perceptions related to physical activity will ultimately affect the choice to engage in physical activity and it is this choice that will affect health outcomes. Health outcomes have been directly associated with affective mood, thus an individual's engagement in physical activity leads to a specific health outcome which cycles back and effects self-efficacy for exercise.

\section{Definitions of Major Concepts}

The expanded conceptual model depicts the proposed relationships among selfefficacy for exercise, religiosity, body image perception (BI), body mass index (BMI), level of education, monthly income, church attendance, residency, age, children, occupation, marital status, and physical activity behavior. All variables included in the model were not addressed in this study but were included for clarity in the conceptualization and discussion of this phenomenon. Pertinent theoretical definitions used in this study are included below.

\section{Physical Activity Behavior}

Physical activity behavior refers to pattern of exercise. In the current study physically active referred to routine engagement in at least 30 minutes of physical activity at least 3 times per week. Physical inactivity referred to engaging in less than 30 minutes of physical activity on less than three days per week. The Stanford Brief 
Activity Survey for Work and Leisure Time Activity (SBAS) was used to measure engagement in physical activity. The SBAS is a 2-item instrument with a 5-point scale. Scale items elicit a summary of an individual's usual amount and intensity of physical activity. The survey represents a modified version of the Stanford Seven-Day Physical Activity Recall (PAR) scale (Taylor-Piliae et al., 2006).

\section{Self-efficacy for Exercise}

Self-efficacy for exercise refers to an individual's personal belief about their ability to routinely engage in physical activity and about personal benefits of routine engagement in physical activity (Bandura, 1997; Schwarzer \& Renner, 2000). The Physical Exercise Self-efficacy Scale (PESES) was used to measure self-efficacy in the current study. Scale items elicit beliefs about personal ability to routinely engage in physical activity. Higher scores represent more confidence in the ability to routinely engage in physical activity while lower scores represent less confidence in ability to routinely engage in physical activity (Brown, 2005).

\section{Religiosity}

Religiosity is a multidimensional construct that refers to an individual's religious worldview. An extrinsic religiosity is a religious worldview that is derived from an external motivation and can be achieved through religious practices. According to Allport and Ross (1967), "the extrinsically motivated person uses his religion"; In contrast, an intrinsic religiosity is a religious worldview that is derived from an internally motivated faith. Moreover, "the intrinsically motivated person lives his religion" (Allport \& Ross, 1967, p. 434). In the current study, intrinsic religiosity is closely related to spirituality.

The God Locus of Health Control (GLHC) scale was used to measure religiosity. Scale items elicit respondents' beliefs about God's control over their health and their personal control over their health. Higher total scores represent the belief that God has more control over their health than the individual and an extrinsic religiosity. Lower total scores represent the belief that personal action has more control over health and an intrinsic religiosity. This questionnaire was chosen because it captures intrinsic and extrinsic dimensions of religiosity, both of which falls within the context of socialcognitive theory and contributes to self-efficacy for exercise.

\section{Body Image}

Body image is defined as the level of satisfaction with one's current body size and shape (Hawkins, Tuff, \& Dudley, 2006). Body image satisfaction varies between African American and Caucasian women. Body image satisfaction has been noted with higher BMI among African American women when compared to Caucasian women (Hawkins et al., 2006). The Appearance Schema Inventory-Revised (ASI-R) was used to measure 
body image perception in the current study. The ASI-R is a Likert-type scale that uses a 5 point rating scale. Higher scores are representative of poorer body image perception and lower scores represent better body image perception.

\section{Residency}

Residency refers to the geographical location of the participant's residence. A crude measure of residency was assessed based on criteria from the Census Bureau (U.S. Census Bureau, 2000). In the current study rural residency referred to areas not serviced by mass transit (i.e., buses) and urban residency referred to areas serviced by mass transit.

\section{Body Mass Index (BMI)}

Body mass index is defined as the relationship of weight (in kilograms) to height (in meters squared) (Green et al., 2006). Body mass index was calculated to determine normal weight, overweight, or obese weight. Height and weight data were collected upon entering the study for this purpose. Guidelines set by the CDC are as follows and were used to categorize participants according to the calculated BMI: 18.5 to $24.9 \mathrm{~kg} / \mathrm{m}^{2}$ as normal weight, 25.0 to $29.9 \mathrm{~kg} / \mathrm{m}^{2}$ as overweight, and $30.0 \mathrm{~kg} / \mathrm{m}^{2}$ and greater as obese (CDC, 2009).

\section{Depressive Symptoms}

Depressive symptoms refer to occasional psychological and physiological feelings similar to those feelings associated with depressive disorders. These feelings can cause sleep disturbance, irritability, and disturbed thought patterns (National Institute of Mental Health, 2009). Examinations of depressive symptoms are relevant in the context of social-cognitive theory as affective mood impacts self-efficacy for exercise. Although depressive symptoms were not measured in this study, a detailed discussion of the impact of this factor on self-efficacy for exercise was included, thus it was appropriate to include an operational definition of this term.

\section{Health}

According to the World Health Organization (WHO), health is a state of complete physical, mental, and social wellbeing, not merely the absence of disease (World Health Organization, 1948). This study indirectly addresses, through discussion, the consequences of each dimension of the WHO definition of the concept of health. This study primarily discussed the obesity-related health complications of diabetes mellitus Type II, coronary artery disease, depressive symptoms, and hypertension. Information regarding the presence of obesity-related health outcomes was not assessed in the current study; however these outcomes are relevant because of the financial and social cost 
associated with these chronic diseases and the subsequent burden to our health care economy.

\section{Support System}

Support system refers to the individuals which serve as a network of encouragement. These persons include spouses, children, friends, fellow parishioners, pastors, and parent figures (Holt et al., 2005). Data related to individual support systems are pertinent within the context of social-cognitive theory and were assessed through information collected on the demographic data form.

\section{Cultural Influences}

Culture refers to behavioral patterns, beliefs, and common practices passed on by way of socialization, hence a cultural influence refers to the impact culture has on the decisions made in life (culture, n.d.). Culture influences perceptions related to religion and body image. This study will examine the influence that common ideologies about religion and body image have on self-efficacy for exercise and physical activity behavior among African American women (Holt et al., 2005).

\section{Significance of Study}

Obesity affects women in African American communities at a greater rate when compared to men and children this population. According to a report from the National Centers for Health Statistic's, 53\% of adult African American women qualified as obese with a body mass index (BMI) of 30 or greater (Ogden, Carroll, McDowell, \& Flegal, 2007). Individuals who are overweight or obese are more likely to develop hypertension and type II diabetes. The prevalence of type 2 diabetes is two to four times higher in African American women than in Caucasian women (Ogden et al., 2007). Additionally, women diagnosed with type 2 diabetes are at greater risk, up to seven times as likely to experience coronary heart disease when compared to women without diabetes. In 2000, about $\$ 117$ billion dollars were spent on direct and indirect obesity-related cost (CDC, 2009).

Within the African American culture women have traditionally held the role of educator, holding strong to religiosity and a sense of oneness with the African American community at large (Wolfe, 2000). The African American woman's position in the social structure within this community places her in an optimal position to influence family members and other members of the African American community. Traditionally, African American women have been portrayed as the domineering head of the household in research literature (Jones, 1992). Although these characteristics have been representative of negative outcomes associated within the culture, the use of a culturally-sensitive approach that builds upon these very characteristics could lead to positive health 
outcomes for African American women, thus having a trickle down effect to the family and population (Jones, 1992).

The use of social-cognitive theory to address physical inactivity in African American women could identify underlying factors that impede engagement in physical activity among this group. Increasing engagement in physical activity among African American women would lead to a decrease in obesity and increase in opportunities for vicarious learning that could lead to an increase in self-efficacy for exercise throughout the entire community. Insight gained through this study could be used to more effectively plan community based participatory research (CBPR) interventions that address pertinent cultural issues and group dynamics in African American communities. Obesity-related complications create a significant financial burden to the healthcare economy each year. Literature notes that African Americans disproportionately experience low socioeconomic status, obesity, obesity-related complications, and physical inactivity. According to the social-cognitive theory the aforementioned factors affect physical activity behavior and should be explored when examining physical activity behavior in African American women. All of these factors exacerbate the growing financial burden on the US healthcare system. Increased physical activity has not been achieved in this community through the research approaches employed in the past because cultural differences have been recognized but inadequately addressed. Past studies have not incorporated approaches to address the impact that culture has on behavior because of the lack of exploration of these relationships. This study attempted to better elucidate the impact that African American church culture has on physical activity engagement through examination of self-efficacy for exercise, religiosity, body image perception, and body mass index among African American women.

\section{Assumptions}

The following assumptions for this study were derived from the framework and study design.

1. There is a difference in the level of physical activity between African American women of faith in the rural community and those in the urban community. Current research suggests that access to venues (i.e., gymnasiums) and structures (sidewalks and playground) for physical activity influences engagement in physical activity. Differences in the availability of structures such as in the urban and rural communities could impact physical activity engagement.

2. Common ideologies that are based on individuals' experience are shared among members in African American churches. These ideologies affect individual perceptions and behaviors. 
3. Women in the African American population can accurately evaluate their selfefficacy for exercise, physical activity engagement, depressive symptoms, and religiosity.

4. Self-report questionnaires will adequately measure psychological phenomena such as perception and religiosity. According to Polit and Beck (2004) it is appropriate to use self-report questionnaires to measure attitudes in adult populations.

5. Participants will honestly disclose there perceptions on the self-report measures.

6. Religiosity is a multidimensional construct that can be accurately assessed using a questionnaire.

7. Self-efficacy for exercise is a multidimensional construct and affected by body image, religiosity, and engagement in physical activity, depressive symptoms, and culture.

8. Women in the African American community have had previous experience with physical activity.

\section{Limitations}

Limitations of this study include the following:

1. The use of self-report and Likert-type scales to collect data and difficulty with isomorphism when assigning value to psychological phenomena is a study limitation. The investigator ensured a safe anonymous environment for participants to promote truthfulness and decrease social desirability response bias (Polit \& Beck, 2004). Selected questionnaires demonstrated reliability and were validated in a similar sample of African American women prior to their use in the current study (Robinson \& Wicks, 2007).

2. This study uses a convenience sample of African American women from a faith community. This approach produces a homogeneous cross section of the African American community. Although the findings cannot be generalized to the other ethnic groups, the findings will be meaningful and will illuminate culture specific factors within demographically similar African American communities and can be generalized to similar samples.

3. The cross-sectional design was appropriate for examination and describing the relationships among the variables in this study of African American women in the church. This study is descriptive and intended to identify associations among 
variables. Additionally, this non-probability sampling approach limits the external validity of the study findings.

4. Quantitative methods were used to explore this psychosocial phenomenon in this study. Investigators exploring emerging psychosocial phenomena may find benefit in taking a positivist view to first explore and describe relationships prior to moving to qualitative methods to further illuminate phenomena (GodfreySmith, 2003). 


\section{CHAPTER 2. REVIEW OF LITERATURE}

\section{Introduction}

Persistent physical inactivity has been an issue for African American women residing in the United States for over a decade. Of particular concern to researchers and healthcare providers alike is the fact that this population disproportionately experiences negative health outcomes, such as obesity, type 2 diabetes and cardiovascular disease when compared to other populations. Published reports substantiate numerous physical and mental health benefits associated with being physically active (Ard \& Rosati, 2000; Ball et al., 2007; Ester et al., 2006; Flynn \& Fitzgibbon, 1998; Franzoni \& Ghiadoni, 2005; McNabb, Quinn, Kerver, Cook, \& Karrison, 1997; Probst et al., 2004; Staffileno et al., 2007). Although, interventions have been implemented with the aim of promoting physical activity behavior among African American women current literature suggest that there has been little sustained impact, as $58 \%$ of African American women continue to report less than recommended physical activity (CDC, 2003; Kim et al., 2006; Wilcox et al., 2007; Yenek et al., 2001).

Researchers have noted the impact of racial/ethnic difference in perception related to body image and religion on behavioral study outcomes such as physical activity. Additionally, differences in socioeconomics such as income and education level have impacted these outcomes as well. Despite recognition of the unique influence African American culture has on perception related to religion and body image few studies have deeply explored these constructs related to physical activity behavior. Moreover, it has been noted that current migration patterns of African Americans could exacerbate physical inactivity among this group. Information obtained in the current study with regard to self-efficacy for exercise, religiosity, body image perception, and physical activity behavior in African American women will facilitate more effective development of culturally tailored intervention designed to promote routine engagement in physical activity.

This chapter will describe in detail, the current state of the literature related to self-efficacy for exercise, religiosity, body image perception, and physical activity behavior. A review of recently published studies relevant to the constructs within the social-cognitive theory model is included in this chapter. An overview of findings from the literature related to self-efficacy for exercise and physical activity behavior, African American culture and body image perception, religiosity and physical activity behavior will be incorporated into the chapter. A summary of the research related to correlates of physical activity and African American women is provided. The chapter concludes with a discussion of how prior studies have informed the current study and a synthesis of the literature. 


\section{Physical Activity}

Physical activity in the basic sense of the matter can best be described as energy expenditure. However, in order to achieve health benefit from physical activity a more intentional approach to this behavior must be employed. The national recommendation for level of physical activity required to produce health benefit is at least 30 minutes of moderate intensity physical activity on most days of the week (National Institute of Health [NIH], 2009). Moderate intensity physical activity is defined as 3 to 6 metabolic equivalents (METs) and can be achieved through various leisure activities such as washing/waxing your automobile (45-60 minutes), gardening (45-60 minutes), pushing a stroller while walking ( $1.5 \mathrm{miles} / \mathrm{min})$ or through organized sports. An important aspect is ensuring that the moderate intensity method chosen is performed for the specified time in order to achieved health benefit.

Research has found that culturally based perceptions related to types of physical activity, time, body image, religion, social support, and socioeconomics impacted physical activity behavior (Davis, Clark, Carrese, Gary, \& Cooper, 2005; Henderson \& Ainsworth, 2000; K. S. Johnson et al., 2005; Jones, 1992; Kim et al., 2006; Yenek et al., 2001; Young, He, Harris, \& Mabry, 2002). Additionally, research has found that confounding effects of demographic factors such as age, educational level, geographical location of residence, and religious practices have influenced exercise behavior in African American women (Dornelas et al., 2007; Probst et al., 2004; Sanderson et al., 2003; Sharma, Sargent, \& Stacy, 2005). Overall affect has been shown to influence physical activity behavior. Depressive symptoms and stress, which may be experienced disproportionately by African Americans, has been shown to be negatively associated with physical activity behavior (Annesi \& Whitaker, 2008; Davis et al., 2005; Jones, 1992; Wise, Adams-Campbell, Palmer, \& Rosenberg, 2006).

\section{Self-efficacy for Exercise}

The aforementioned constructs contribute to individual self-efficacy for exercise, the core concept within the social-cognitive theory model and a major focal point of this study. Self-efficacy for exercise has been shown to be positively associated with exercise intention (Schwarzer \& Renner, ND). However, various studies conducted in the African American female population have used the social-cognitive theory as the guiding framework for their design and have used self-efficacy for exercise as an outcome evaluation with inconsistent results as to the association of this concept and physical activity behavior (Ball et al., 2007; Dornelas et al., 2007; Young \& Stewart, 2006). Although African American women have been found to have a high degree of selfefficacy for exercise across all reported levels of physical activity some studies report no association between physical activity behavior and self-efficacy for exercise (Felton, Boyd, Bartoces, \& Tavakoli, 2002; Sanderson et al., 2003). Other studies report a negative association between self-efficacy for exercise and physical activity behavior. Researchers have offered explanations such as, African American women being over confident in their ability to engage in routine physical activity based on prior experience 
with physical activity, for this outcome (Wilber et al., 2003). Other researchers report a positive association between self-efficacy for exercise and physical activity behavior (Ball et al., 2007; Fallon, Wilcox, \& Ainsworth, 2005; Sniehotta, Scholz, \& Schwarzer, 2005; Young \& Stewart, 2006). In addition to the previous explanation cited the resulting inconsistency could be related to other factors such as methods used to measure outcomes and length of intervention. A detailed discussion of these methodological issues will be included in the synthesis of literature portion of this chapter.

\section{Socioeconomic Factors and Self-efficacy for Exercise}

According to the social-cognitive theory, which is the framework for this study, self-efficacy for exercise is mediated by several factors including body image perception, BMI, support systems, educational level, income, place of residency, and perceptions related to religion. Additionally, home making responsibility, raising children and being employed could influence a woman's affect and her choice to engage in physical activity. Many studies have found that the value placed on personal appearance contributes significantly to body image perception a correlate of self-efficacy for exercise (Altabe, 1998; Breitkopf \& Littleton, 2007; Cash, Fleming, Alindogan, Steadman, \& Whitehead, 2002; Rubin, Fitts, \& Becker, 2003). Although body image perception has been positively associated with physical activity behavior in other populations this relationship has not been confirmed in African American women (Davis et al., 2005; Hawkins et al., 2006; Young et al., 2004; Young et al., 2002). The most commonly cited explanation for the inconsistent findings related to body image perception and physical activity in African American women have been culturally based. Perceptions related to weight commonly measured and referred to as BMI has been associated with physical activity behavior (Felton et al., 2002). Studies have found that African American women report a more positive body image at higher BMI's when compared to other populations (Altabe, 1998; Breitkopf \& Littleton, 2007; Davis et al., 2005; Hawkins et al., 2006; Miller et al., 2000; Young et al., 2002). This could explain the conflicting association noted among intervention studies focused solely on physical activity for weight reduction in African American women.

Research suggests that adequate social support from friends and families facilitate physical activity behavior (Fleury, Harrell, \& Cobb, 2001; Henderson \& Ainsworth, 2000). These women expressed that having a companion to exercise with provided companionship and, motivation as well as a role model for engaging in physical activity. These findings are both supportive of and relevant to the social-cognitive theory (Bandura, 1994; Gecas, 1989). Additionally, having support from families could reduced perceived guilt associated with taking time from family responsibility, which has been cited as a barrier to engaging in physical activity (Henderson et al., 2000; Nies et al., 1999; Young et al., 2002). Studies have also shown that the African American population has reported greater participation on religious activities such as church attendance, when compared to Caucasians (Sanderson et al, 2003). According to the 2003 Behavioral Risk Factor Surveillance System (BRFSS), 85\% of the women in Alabama reported attending religious services. Various church and faith-based studies have cited that for African 
American women, this increased church attendance could provide opportunities for forming valuable social networks which have been noted to mediate self-efficacy for exercise (Sanderson et al, 2003). Selection processes in the social-cognitive theory reveal environmental correlates of physical activity which mediate self-efficacy for exercise.

Engaging in physical activity is not only based on intention and action, but also on availability of venues for activity. Women in the African American community have cited economic disparities, lack of access to facilities and unsafe neighborhoods as barriers to physical activity (Nies et al., 1999; Young et al. 2002). Reports indicate that African American populations are migrating to rural areas which may not provide convenient access to venues for physical activity (Probst et al., 2004; Sallis, Hovell, \& Hofstetter, 1992; Scharff, Homan, Kreuter, \& Brennan, 1999). Concerns over safety related to poorly lit streets and roaming dogs could hinder engagement in physical activity for rural dwelling African American women. Reports of socioeconomic status of rural dwelling African Americans suggest overall lower educational level (Probst et al., 2004). Educational level, income level, marital status, and number of children are a reflection of one's personal choices and affect self-efficacy for exercise through selection processes in the social-cognitive theory. Of these variables age, income and level of education have been noted as having a confounding effect on physical activity behavior (Ball et al., 2007; Clark \& Nothwehr, 1999; Richter, Wilcox, Greaney, Henderson, \& Ainsworth, 2002; Sanderson et al., 2003; Wise et al., 2006). Although marital status and number of children have not been directly associated with physical activity behavior they have been cited in the literature as being associated with mood (Annesi \& Whitaker, 2008; Grote, Bledsoe, Larkin, Lemay, \& Brown, 2007; Henderson \& Ainsworth, 2000; Wise et al., 2006). Affective processes such as depressive symptoms mediate selfefficacy for exercise. The current study does not measure depressive symptoms; however number of children and marital status were assessed and will be considered in reference to overall level of self-efficacy for exercise for discussion at the conclusion of the study.

\section{Body Image Perception and African American Culture}

The research community has documented a general consensus that the African American community in general exhibit a more positive body image and an overall acceptance of heavier body sizes or BMI when compared to other populations (Cash, Melnyk, \& Hrabosky, 2004; Davis et al., 2005; Hawkins et al., 2006; Miller et al., 2000; Young et al., 2004). Numerous explanations for this prevalent perception within the culture have been offered and will be reviewed here in an effort to better understand how African American culture informs individual body image perception. Historically, African Americans have associated excess body weight with better health, wealth, and even fertility (R. W. Johnson \& Broadnax, 2003). Biological perspectives on African American perceptions and weight have been one that associated cultural weight disparities with an innately larger musculoskeletal system (Rubin et al., 2003). This cultural appraisal of excessive weight has been cited as explanatory for the positive association between higher BMI and less body image disturbance among members of the African American population (Altabe, 1998; Breitkopf \& Littleton, 2007; Cash et al., 
2002; Celio, Zabinski, \& Wilfley, 2002; Thomas, Moseley, Stallings, Nichols-English, \& Wagner, 2008). Although this positive outlook on body image fosters psychological resilience it has been associated with devaluing physical activity behavior in a population that disproportionately experience obesity and obesity-related complications (Breitkopf \& Littleton, 2007; Jacobson, Morton, Jacobson, Sharma, \& Garcia, 2002; Thomas et al., 2008).

\section{Body Image Perception and African American Women}

To say that evaluation of body image perception in African American women is complicated would be a gross understatement. Mainstream culture has typically defined and evaluated body image perception on over all thinness. Society and media bombard the senses with this imagery that is not representative of the vast diversity of the world. This cultural misrepresentation is cited as leaving ethnic minorities such as African American women feeling oppressed and marginalized (Ruben et al., 2003). Research suggests that African American women have adapted a more holistic view of beauty that extends beyond the physical body to include attitude, style, and personality (Flynn \& Fitzgibbon, 1998; R. W. Johnson \& Broadnax, 2003; Rubin et al., 2003). Although this adaptation is of psychological benefit (i.e., protective effect against onset of depression) to this population, poor understanding and incomplete assessment of this factor by the research community could be detrimental to the physical health by decreasing the overall effectiveness of exercise interventions designed for African American women (Wise et al., 2006). Cash et al. (2002) has found that body image is multidimensional with both weight and non-weight related dimensions.

Several studies have found that African American women report a more positive body image perception at higher BMI's than other populations (Altabe, 1998; Breitkopf \& Littleton, 2007; Cash et al., 2004; Celio et al., 2002; Miller et al., 2000; Roberts, Feingold, Cash, \& Johnson, 2006; Thomas et al., 2008). In contrast, other studies have revealed a negative association between body image and BMI (Davis et al., 2005; Pumariega, Gustavson, Gustavson, Motes, \& Ayers, 1994). The inconsistencies most likely reflect flaws in measurement. According to Cash et al. (2004), African American women place less value on the physical body with respect to beauty, thus evaluation of body image employing such measures could produce false representation of this perception. Motivational salience refers to the determinants of one's cognitive-behavioral investment (Cash et al., 2004). African American women exhibit equivocal levels of motivational salience when compared to Caucasian women (Roberts et al., 2006). However, few studies have evaluated body image perception with respect to physical activity behavior in African American women with inconsistent results. McNabb et al. (1997) utilized a design approach that promoted physical activity to improve health rather than to lose weight in an effort to minimize the effect of culturally based body image perceptions. The use of the aforementioned culturally-sensitive design approach recognized that this culture places more value of heath than on weight. Although no between-group difference in physical activity was noted, the 14 week church-based intervention study documented weight loss and improved health (Miller et al., 1998). 
Women in general have been shown to invest time and effort in to their appearance, however culturally influenced differences in individual perceptions of beauty (i.e., overall physical, anatomical features, hair style, dress) will need to be carefully considered when tailoring interventions and choosing methods of measurement (Cash et al., 2004; Kumanyika \& Charleston, 1992; Roberts et al., 2006).

\section{Religiosity and African American Culture}

Historically African American culture has fostered using religion as a coping strategy, a practice that has been attributed to their experience during slavery (Black, 1999; Holt et al., 2005). During slavery, elderly African American women in their dealing with the pain and mental anguish of estrangement from loved ones and physical abuse, offered prayers to God in reciprocity for mercy and relief from suffering (Black, 1999; Jones, 1992). This behavior provided a means of reframing harsh circumstances while maintaining a sense of self worth during the time of slavery. Today members of the African American population continue to experience the hardship of poverty, marginalization, and health disparities, thus prayer as a means of coping has become a tradition in this culture (Holt et al., 2005).

African Americans, compared to other populations report greater participation in organizational and non-organizational religious activities (Chatters, Levin, \& Taylor, 1992; K. S. Johnson et al., 2005; Milevsky \& Levitt, 2004). The level of participation in these activities may be associated with chronic stress brought on by a perceived history of oppression and mistreatment, or acute stress associated with illness, economic hardship and care giving responsibilities (Ark, Hull, Husaini, \& Craun, 2006). Going to church seems to be a well accepted coping mechanism and offers a means of escape from the daily stressors of life such as racism, care giving obligations and financial concerns (Holt et al., 2005). Members of the African American community often find refuge within the African American church and view the church as a place to openly express themselves and receive emotional support (Ark et al., 2006; Chatters et al., 1992). There are mixed results as to the effects of religiosity on health behaviors (Johnson, et al., 2005; Milevsky \& Levitt, 2004).

The vast majority of the studies have found a positive association between religiosity and health promotion behavior (Ai, Peterson, Rodgers, \& Tice, 2005; Payne, Bergin, Bielema, \& Jenkins, 1991). Other studies report a negative association between religiosity and health promotion behaviors (Kinney, Emery, Dudley, \& Croyle, 2002; Koenig, Moberg, \& Kval, 1988; Wallston et al., 1999). Others report no relationship between religiosity and health (Atchley, 1997). Religiosity has been used interchangeably with religious coping and is noted as a multidimensional construct (Milevsky \& Levitt, 2004; Moshki, Ghofranipour, Hajizadeh, \& Azadfallah, 2007; Pargament, 1997; Wallston et al., 1999). The varying ways that this construct is being used could account for inconsistent findings in the literature. Intrinsic and extrinsic religiosity exerts different effects on the psyche (Milevsky \& Levitt, 2004; Pargament, 1997). Studies have shown that African Americans experience a higher degree of religiosity and a religious locus of 
control that placed more responsibility for their health and illness on God as a means of coping (Johnson et al., 2005; Pargament, 1997). Although several investigators have examined the role of religious beliefs with regard to understanding, coping with, and recovering from illness, few studies have focused on the role of religious beliefs related to health promotion and disease prevention in African American adults (K. S. Johnson et al., 2005; Kinney et al., 2002).

\section{Religiosity and African American Women}

Women in the African American population have traditionally held the role of educator, holding strong to religiosity and a sense of oneness with the African American community at large (Jones, 1992; Wolfe, 2000). The aforementioned statement has been partially recognized in several studies that have noted that African American women participate in religious activities such as going to church more frequently than men and other populations (Green et al., 2006; Sanderson et al., 2003; Sharma et al., 2005). Studies have found that in general African American women view their religiosity in terms being of health protective (Banks-Wallace \& Parks, 2004; Matthews, Lannin, \& Mitchell, 1994; Wilson-Ford, 1992). However confounding factors related to demographics, socioeconomics and family or caregiver responsibilities have been cited as potential barriers to such health protective behaviors as routine engagement in physical activity.

Studies have cited that African American women are often single parents and/or assume caregiver roles (Chadiha, Adams, Biegel, \& Gutierrez, 2004; Dornelas et al., 2007; Sharma et al., 2005; Wallace-Williams, Dilworth-Anderson, \& Goodwin, 2003). Moreover a negative association has been noted in African American women between being partnered and engaging in physical activity (Sanderson et al., 2003). Additionally, studies have noted socioeconomic disparities between African American women and other populations. Reports have noted lower education and income levels among African American women when compared to other populations (Ball et al., 2007; Probst et al., 2004; Sanderson et al., 2003, Young et al., 2002). These factors coupled with the knowledge that African American women have been noted as having the perception that African American culture places additional responsibilities on them when compared to Caucasian women could have significant effects on overall affective mood, a contributory component to self-efficacy for exercise (Bandura, 1994; Gecas, 1989). Many studies conducted in the African American female population have been implemented through the Black church (Dornelas et al., 2007; Kim et al., 2006; Kumanyika \& Charleston, 1992; Yenek et al., 2001; Young \& Stewart, 2006). Although attrition has been noted as problematic with respect to this approach, it seems feasible that reach within the African American female population can be achieved in this manner. 


\section{Physical Activity, Religiosity, and African American Women}

While several studies have been conducted with the aim of increasing physical activity among African American women for more than a decade, physical inactivity remains an issue in this population (Dornelas et al., 2007; Kim et al., 2006; Kumanyika \& Charleston, 1992; McNabb et al., 1997; Wilcox et al., 2007; Yenek et al., 2001; Young \& Stewart, 2006). Research supports culturally-sensitive approaches to health promotion interventions (Resnicow et al., 1999). Several studies have recognized that religion is a central part of African American culture and have targeted subjects in the African American church as an approach to meet this need (Kim et al., 2006; Kumanyika \& Charleston, 1992; McNabb et al., 1997; Wilcox et al., 2007; Yenek et al., 2001; Young \& Stewart, 2006).

Study design issues and high attrition rates have made it difficult to modifying research approaches to strengthen interventions that have focused on health promotion in African American populations. Sentinel church-based studies noted intervention success citing participants achieved significant weight loss (Kumanyika \& Charleston, 1992; $\mathrm{McNabb}$ et al., 1997). These studies focused on diet and exercise to promote health. Although the researchers documented weight-loss in the sample, they reported little or no data related to the physical activity outcomes of the study; hence it is difficult to determine which variable accounted for the change in weight. Kumanyika and Charleston (1992) conducted their 8 week single-group delayed intervention study in a sample of moderately overweight African American women $(N=187)$ and noted a $41 \%$ attrition rate. Moreover, McNabb et al. (1997) conducted their 14 week randomized betweengroups intervention study in a very small sample $(N=39)$. Subsequent church-based studies have indicated intervention success, however not solely based on increased physical activity. Dornelas et al. (2007) conducted 10 week aerobic exercise intervention in a relatively small sample $(N=76)$ of urban ethnic minority women (African American women $=46 \%$ ) and based intervention success on session attendance rather than physical activity. Additionally, the investigators cited a $43 \%$ attrition rate. Latter church-based studies noting intervention success have been conducted over longer time periods and included larger samples. Young and Stewart (2006) conducted their 6 month randomized between-groups aerobic exercise intervention in a larger sample of African American women $(N=196)$ and noted a decrease in the prevalence of physical inactivity in both groups. This study addressed cultural issues in their study by employing a CBRP approach and used the social-cognitive theory framework as a guide. Although intervention success was noted researchers noted low attendance in the follow-up phase. Although the CBPR approach used in the planning of this study contributed to positive outcomes attrition hinder follow-up biophysical assessment that could have substantiated the effects of change in reported physical activity.

Research has recognized that simply implementing intervention through the church (church-based) may not fully mediate the effects of culture in this population (Resnicow et al., 1999). Although church-based intervention aided in the recruitment aspect of the studies, retention which is essential for sustained impact remains an issue. In an effort to gain deeper insight into the African American culture, researchers conducting 
studies through the African American church have begun to use a faith-based rather than church-based approach. Few large scale faith-based studies have been implemented through the African American church with mixed results on physical activity outcomes (Kim et al., 2006; Wilcox et al., 2007; Yenek et al., 2001). Wilcox et al. (2007) conducted (Healthy-AME) a large scale $(N=889), 3$ year randomized between-groups intervention study. This collaborative research effort between the noted principle investigators, the Medical University of South Carolina, the University of South Carolina and the $7^{\text {th }}$ Episcopal District of the African Methodist Episcopal Church was designed using the CBPR approach. The AME church was established in 1787 by Bishop Richard Allen and other freed slaves, thus collaboration with this institution provided a rich opportunity to gain deep insight into African American culture and history. The social ecology and transtheoretic Models were used as theoretical framework for this study. These investigators found that health information awareness, along with fruit and vegetable consumption increased over the two-year period of the study but physical activity did not. However trend data suggested change in the social attitude towards physical activity with reports of increased encouragement from church members to engage in physical activity. This is a very significant and relevant point to the proposed study in that studies cited throughout this chapter have noted that African American women cited social support from friends and family as facilitators to physical activity.

Project Joy, conducted by Yenek et al. (2001) was a large scale $(N=529)$ randomized nutrition and activity intervention study. This study (Project Joy) was aimed at reducing cardiovascular risk factors in African American women. Although most of the program outcomes were achieved there was no significant increase in physical activity. The authors noted a substantial decrease in attendance in their volunteer led intervention group in comparison to the staff led group and found that participants did not feel comfortable being advised by their peers (Yenek et al., 2001). Additionally, overall lack of interest in the contingent self-help group was suggestive of this population's religious perception. In contrast to the other faith-based studies cited, the WORD (Wholeness, Oneness, Righteousness, Deliverance) study was an eight week behaviorally focused weight loss study which focused on diet and exercise (Kim et al., 2006). Although participants reported increased recreational physical activity, the change in behavior was small in comparison to other church-based physical activity studies (Kumanyika \& Charleston, 1992; McNabb, 1997). Additionally, these investigators only reported recreational physical activity despite the fact that intensity and duration of physical activity should be considered when establishing cardiovascular benefit.

\section{Summary of the Review of Literature}

Several studies through many disciplines have noted the complexities related to behavior among African American women. However few have been able to document sustained increase in physical activity behavior. In the spirit of scientific inquiry, it seems that researchers have taken a more Positivist view towards exploring behavior. However, noted cultural complexity warrants a more holistic view of this phenomenon at this point. Hence the need for a more comprehensive approach addressing culturally relevant factors 
such as body image perception, socioeconomics, and religion to research design related to physical activity behavior in African American women. Disparities in income, education, residency, perceptions of caregiver responsibilities, and religious schemas have all been noted to affect health behaviors in African American women. Additionally, the affects of differing perceptions of beauty and motivation related to physical activity in this population should be explored. Studies have shown that African American women often reject the mainstream culture's definition of beauty and perceive beauty in terms of style (i.e., hairstyles, skin color, and attire) and presence in addition to body size (i.e., BMI). Using the social-cognitive theory as its framework the proposed study examined selfefficacy for exercise, religiosity, body image perception and BMI with respect to physical activity behavior. 


\section{CHAPTER 3. METHODOLOGY}

This chapter outlines a detailed discussion of the research methodology including the study design chosen to answer the research questions presented in Chapter 1. Specific details related to the sample, setting, instrumentation, procedures, statistical analyses, and measures used to protect human subjects are included.

\section{Research Design}

This cross-sectional, descriptive, comparative study was used to explore exercise behavior among African American women. According to the social-cognitive theory, it is appropriate to use the cross-sectional approach to examine the effects of culturally influenced perceptions (self-efficacy for exercise, religiosity, and body image) on behavior (engagement in physical activity). A descriptive approach was used to characterize a single sample of African American women. There is limited in-depth data related to exercise behavior in African American women. The principal investigator (PI) chose this approach as a logical starting point for illuminating the complexities surrounding persistent physical inactivity among African American women. A comparative design was incorporated into the study to distinguish group differences between participants who attended church services frequently versus those who did not. Additionally, differences between participants who reside in rural versus urban areas were investigated.

\section{Setting and Sample}

This study included a convenience sample of 100 African American women recruited through an African Methodist Episcopal church in a large urban city and through various community contacts established during this initial data collection session. The researcher planned to recruit African American women through multiple African American churches, universities, local YMCAs, and public parks; however after the first data collection session the recruitment phase took a grass roots turn with participants organizing groups of friends, family, and coworkers to participate in the study. Four additional data collection sessions followed taking place in an area rehabilitation clinic and the personal residence of the participants. The PI was notified by phone of the number of interested women and the location once the groups were organized. The researcher arranged data collection times that ranged from mid-morning to late evening, to make involvement convenient for participants.

The inclusion criteria for participation in the study are included below.

1. Self identified as an African American female

2. Between the age of $21-60$ years

3. Able to read and/or understand English at the $6^{\text {th }}$ grade level 
4. Willing to complete the 1.5 hour study requirements

To ensure adequate sample size and external validity, 100 African American women were recruited for this study. Power analysis was performed prior to conducting the study to determine sample size requirements to ensure a p-value less than or equal to .05 using the statistical package Sample Power 2.0. The effect size was chosen according to Cohen's criteria (1988) for a small effect size. For multiple regression analyses, a small effect size of .15 was used. This number represents the smallest effect size that would indicate a significant association (Cohen, 1988). According to Polit and Beck (2004), typical associations between psychological variables range from .10 to .40. Independent variables were chosen and entered into the regression model based on published research findings and the strength and association with the dependant variables. Categorical independent variables were dummy coded, where the absence of a characteristic was coded 0 and presence of a characteristic was coded 1, prior to conducting regression modeling. Alpha was set at $\mathrm{p} \leq .05$ for all statistical analyses, yielding a power of .70 .

\section{Instrumentation}

In this study, five paper and pencil questionnaires that focused on the study variables included in the research questions were given to participants as they entered the study. In addition to completing the questionnaires, participants were asked to complete a standardized demographic data form. The PI was available during completion of the forms and provided assistance to participants as needed. Written permission and/or proof of purchase were obtained for the instruments used in this study (Appendix A). The following self-administered instruments were used: The Standardized Demographic Data Collection Form, Physical Exercise Self-efficacy Scale (PESES), God Locus of Health Control (GLHC), Stanford Brief Activity Survey (SBAS), and the Appearance Schema Inventory - Revised (Brown, 2005; Cash et al., 2004; Taylor-Piliae et al., 2006; Wallston et al., 1999).

\section{Demographic Data Collection Form}

The standardized demographic data collection form was developed to capture demographic characteristics of each participant (Appendix B). The PI developed this self administered form. Each participant was asked to provide information such as age, marital status, ethnicity, gender, monthly income, and number of children. Flesch Kincaid assessment indicated the reading level of the standardized demographic data form was 6.5. Estimated time to complete this form was 15 minutes. 


\section{Physical Exercise Self-efficacy Scale}

The Physical Exercise Self-efficacy Scale (PESES) was used to measure selfefficacy (Appendix C). The PESES was developed by Schwarzer and Renner (ND) to measure confidence in one's ability to adhere to an exercise program. The PESES is a 5item Likert-type scale that uses a rating of 1 to 4; a response of 1 reflects "very uncertain" and 4 reflects "very certain". Scale items elicit beliefs about personal ability to routinely engage in physical activity. The total score reflects the sum of all the items and ranges from 5 to 20 . Higher scores represent more confidence in the ability to routinely engage in physical activity while lower scores represent less confidence in ability to routinely engage in physical activity. In this study total scores that ranged from 5 to 12 indicated less confidence in exercise intention and total scores that ranged from 13 to 20 indicated more confidence in exercise intention.

This scale is a valid measure of self-efficacy for exercise, the focus of socialcognitive theory. Reliability and validity testing of this instrument has been conducted in a variety of samples. Internal consistency in a large community sample $(N=1,722)$ was reported as .88 . Scale scores were significantly and moderately correlated with exercise intention $(\mathrm{r}=.33, \mathrm{p}<.01)$ and physical activity behavior $(r=.39, \mathrm{p}<.01)($ Schwarzer $\&$ Renner, ND). The scale significantly and moderately correlated with physical activity behavior $(\mathrm{r}=.29, \mathrm{p}<.05)$ in a sample of female Caucasian college students (Brown, 2005). The scale was validated in a pilot study in a sample of African American females $(N=19)$ and exhibited adequate internal consistency reported as .80 (Robinson \& Wicks, 2007). Results in this pilot study also suggested a weak positive correlation between selfefficacy for exercise and GLHC $(r=.36, p \leq 05)$.

Limitations of the PESES are that current evidence of generality of the scale across all age groups has not been established. The age of participants in the current study ranged from 21 to 60 years. Additionally, age was examined as a correlate of selfefficacy for exercise in this study. The Flesch-Kincaid assessment of the PESES indicated a reading level of 8.0. The approximate time for completing the PESES is 10 minutes.

\section{God Locus of Health Control}

The God Locus of Health Control (GLHC) scale was used to measure religiosity (Appendix D). The scale is a modification of the general form of the Multidimensional Health Locus of Control scale that was designed to assess various types of control related cognitions (Wallston et al., 1999). The GHLC was developed by Welton et al. (1996) to assess religiously based health control. The self-report instrument has 6 items designed to assess respondents' beliefs about God's control over their health versus their personal control over their health. The GLHC is a Likert-type scale that uses a rating of 1 to 6 with 1 indicating that they "strongly disagree" and 6 indicating that they "strongly agree" with items within the scale. The total score reflects the sum of all the items and ranges from 6 to 36. Higher total scores represent the belief that God has more control over their health 
than the individual and an extrinsic religiosity. Lower total scores represent the belief that personal action has more control over health and an intrinsic religiosity. In the current study total scores that ranged from 6 to 15 indicated an intrinsic religiosity that placed less responsibility for one's health on God and total scores that ranged from 16-36 indicated an extrinsic religiosity that placed more responsibility for one's health on God.

Previous studies that included individuals from other ethnic groups suggest that this tool is a valid measure of religious beliefs about control over health and has excellent internal reliability with a Cronbach alpha of .90 (Murry et al., 2006; Wallston et al., 1999). Additionally, other investigators have found that extrinsic religiosity is positively associated with participation in religious activities and negatively associated with health protective behavior (Holt et al., 2005; Koenig, et al., 1988; Kinney et al., 2002; Wallston et al., 1999). In a sample of individuals with rheumatoid arthritis $(N=145)$ the GLHC was moderately correlated with participation in religious activities $(r=.32, p<.01)$ suggesting criterion-related validity. In a study evaluating screening behaviors among African American women $(N=52)$ researchers found that increased scores on the GHLC were inversely related to breast cancer screening and mammography citing that each onepoint increase in the GLHC resulted in a $12 \%$ reduction in likelihood towards screening behavior (Kinney et al., 2002) suggesting criterion-related validity of this instrument. This questionnaire was chosen because it captures intrinsic and extrinsic dimensions of religiosity, both of which fall within the context of Social Cognitive theory as contributing factors to self-efficacy for exercise. The Flesch-Kincaid assessment of the GLHC indicated a reading level of 6.9. The approximate time for completion of the GLHC is 10 minutes.

\section{Stanford Brief Activity Survey}

The Stanford Brief Activity Survey for Work and Leisure Time Activity (SBAS) was used to measure engagement in physical activity (Appendix E). The self-report instrument has 2 items which elicit a summary of the usual amount and intensity of physical activity. The SBAS was developed by Taylor-Piliae et al (2005) and represents a modified version of the Stanford Seven-Day Physical Activity Recall (PAR) scale. The first item on the questionnaire describes different types of on-the-job activity and the second item describes different types of leisure time activity. Each item has five response choices that include detail about frequency, intensity, time, and types of activity. The instrument is a Likert-type scale in that each question requires the participant to select one statement that best describes their usual activity. Each response to the two items represents more frequent and vigorous activity with the first response reflecting "inactivity" and the last response reflecting "very hard intensity". A SBAS score indicating physical activity at moderate or greater intensity had a sensitivity of .75, a specificity of .58 , and was able to detect nationally recommended physical activity levels of 150 minutes or more per week in a sample of active older adults $(N=1,010)$. Additionally, these researchers found that individuals in higher activity categories had more favorable cardiovascular disease risk factor profiles including a lower BMI. In a study of older adults, face and content validity was established by two investigators 
separately grading each response category using established metabolic criteria. Inter-rater reliability was established through a comparison of the independent scoring categories which resulted in a scoring mechanism representing the five different activity categories on the instrument; however no inter-rater correlation coefficient was documented on the SBAS. Composite scores on the SBAS range from 1 representing "physical inactivity" to 5 representing "routine engagement in very hard intensity physical activity". In this study total scores that ranged from 1-2 indicated physical inactive and total scores that ranged from 3-5 indicated being physically active.

Limitations of the SBAS include those associated with self-report measures and that it does not provide detailed information about patterns of activity (Taylor-Piliae et al., 2005). Results from the SBAS reflect usual physical activity over the span of a year and consideration should be given to possible variations due to special circumstance such as acute illness. Additionally, the instrument can appear complex to participants because of the wordiness of each item. Detailed verbal instruction for completing this instrument was given to each participant in addition to the written instructions included on the questionnaire. This instrument was selected because of its documented reliability, concurrent validity with the Stanford Seven Day PAR instrument $(\mathrm{p}<.01)$, and brevity. Additionally this instrument is relevant within the social-cognitive theory framework. A Flesch Kincaid assessment of the SBAS indicated a reading level of 10.0. The approximate time for completion of the SBAS is 15 minutes.

\section{Appearance Schema Inventory - Revised}

The Appearance Schema Inventory - Revised (ASI-R) was used to measure body image perception in this study (Appendix F). Cash and Labarge developed the ASI-R, a revised version of the Appearance Schema Inventory in 1996 (Cash et al., 2004). This revised version was developed to overcome noted limitations related to inclusion of items that detailed social stereotypes, inadequate number of items to assess behavior, and the inability to discriminate gender difference in the original Appearance Schema Inventory. The resulting ASI-R assesses an individual's body-image investment vis-àvis certain beliefs about their own appearance (Cash et al., 2004). This self-report measure has 20 items that focus on six domains that influence body image perception including historical salience, attentional and cognitive salience, self awareness, behavioral, affective, and interpersonal salience. These domains are encompassed in two sub-scales, termed Self-Evaluative Salience (12 items) and Motivational Salience of Appearance ( 8 items). Six of the 20 items are negatively worded and require reverse scoring to address response set issues. The ASI-R is a Likert-type scale that uses a 5 point rating scale with 1 indicating respondents "strongly disagree" and 5 indicating that they "strongly agree" with the item. The composite score is the sum of all items divided by the number of items and can range from 1 to 5 . Higher scores are representative of poorer body image perception and lower scores represent better body image perception. In this study total scores that ranged from 1.0-2.5 indicated better body image perception and total scores that ranged from 2.6-5.0 indicated poorer body image perception. 
Reliability and validity testing of this instrument has been conducted in a multiple diverse samples of college students including both men and women Caucasian and African Americans (Cash et al., 2004). Internal consistency in these samples ranged from .82 to .90 . The ASI-R demonstrates significant and moderate convergent validity with the original Appearance Schema Inventory $(r=.76, p<.00)$ but with improved psychometric properties. Additionally, convergent validity among female respondents is noted with the following body image measures: Body-Image Ideals Questionnaire $(\mathrm{r}=$ $.53, \mathrm{p}<.00)$, Situational Inventory of Body-Image Dysphoria $(\mathrm{r}=.67, \mathrm{p}<.00)$, and Multidimensional Media Influence Scale-Internalization Subscale $(r=.64, p<.00)$. A Flesch Kincaid assessment of the ASI-R indicated a reading level of 7.0. The approximate time for completion of the ASI-R is 20 minutes.

The PI used one scale and tape measure to collect all body measures. The same household-type scale and tape measure was used to collect height and weight on each participant. The scale was manually calibrated during setup prior to each data collection session to ensure accuracy. The tape measure was secured to the wall with tacks. Participants remained fully dressed with the exception of their shoes while heights and weights were obtained.

\section{Procedure}

Permission to conduct the proposed study was obtained from the University of Tennessee Health Science Center's Institutional Review Board (IRB). A letter of approval from the IRB was provided classifying the study as an exempt review (Appendix G). Verbal and written approval was obtained from the pastor of the participating AME church to invite African American women to participate in the study (Appendix H). The PI collected all study data.

The PI addressed the congregation during the Sunday worship service to briefly introduce the study, outline inclusion criteria, and invite members to participate. Interested participants were instructed to come to the fellowship hall of the church immediately following services for more information and to participate. Interested participants were greeted by the PI as they entered the fellowship hall, questions were entertained, and the age criterion was verbally verified. Interested participants attended data collection sessions at either a personal residence or in a break area as designated by the group's organizer. Participants' were greeted by the PI with a brief introduction to the study and a review of the inclusion criteria. The group was given an opportunity to ask questions during the data collection sessions. The PI instructed all persons between the ages of 21 and 60 to raise their hands as verification of meeting the age criterion. Participants meeting the criteria were given a pencil along with the study packet containing an informed consent document (required for exempt studies) summarizing the research study and the 5 questionnaires. Prior to being directed to a seating area to complete the questionnaires height and weight measurements, to be used for calculating BMI, were obtained and recorded on the inside of the back cover of the packet. 
Once questionnaires were completed and returned, the PI reviewed the participant's responses. If missing data were present the PI brought it to the attention of the participant and provided an opportunity for them to complete the data requested. Participants were given a $\$ 20$ dollar gift card once completed study packets were returned. All study packets were numerically coded to protect the participants' confidentiality and for organization of the data set.

\section{Site Preparation}

Separate areas for completion of questionnaires and obtaining height and weight on the participants were set up. The same household type scale and tape measure was used to collect height and weight on participants. This material was setup in the designated area by the PI upon arrival to the site. The scale was manually calibrated during setup prior to each data collection session. The designated area, which allowed for privacy for the participant, was a classroom in the fellowship hall, a bathroom in personal residences, and an unused treatment area in the rehabilitation clinic. Refreshments were made available in the area designated for completion of questionnaires during two data collection sessions. Additionally, refreshments were only provided when the person organizing the groups (not the PI) suggested, coordinated and served them. The PI did not oppose this practice as the person organizing the group volunteered to provide refreshments as an additional incentive to participants.

\section{Data Analysis}

The following statistical tests were conducted to describe this study's sample and to answer study research questions. The corresponding aim and research question a presented prior to outlining the data analyses.

\section{Analysis of Sample Characteristics}

It is appropriate to obtain descriptive statistics to describe the demographic characteristics of this sample of African American women prior to answering the research questions. Measures of central tendencies and distribution characteristics were obtained for each study variable.

\section{Specific Aim 1}

Explore the relationships among self-efficacy for exercise, religiosity, body image perception, body mass index, socioeconomic factors (i.e., level of education, monthly income, church attendance, residency [i.e., rural or urban], age, parental responsibility [i.e., caring for children under age 16], number of children, occupation and marital status), and physical activity behavior among African American women. 
Research Question 1: What are the relationships among self-efficacy for exercise, religiosity, body image perception, body mass index, level of education, monthly income, church attendance, residency (i.e., rural or urban), age, parental responsibility (i.e., caring for children under age 16), number of children, occupation, marital status, and physical activity behavior among African American women?

Prior to calculating correlation coefficients scatter plots were run to screen the data for evidence of linearity and for outliers. Additionally, a normal log-transformation was performed on data with marked deviations from normality (i.e., skewness value $>-.4$ or < .4). Pearson's product-moment tests were performed to assess correlations among interval/ratio level data. Spearman's rho tests were performed to assess correlation among ordinal data.

\section{Specific Aim 2}

Compare the level of self-efficacy for exercise, religiosity, body image perception, body mass index, socioeconomic factors (i.e., level of education, monthly income, church attendance, residency [i.e., rural or urban], age, parental responsibility [i.e., caring for children under age 16], number of children, occupation and marital status), and physical activity behavior among African American women that regularly attend church services (at least 3 times out of the month) to those who do not regularly attend church services (less than 3 times out of the month).

Research Question 2: What are the differences in self-efficacy for exercise, religiosity, body image perception, body mass index, socioeconomic factors and physical activity behavior among African American women that regularly attend church services (at least 3 times out of the month) compared to those who do not regularly attend church services (less than 3 times out of the month)?

Independent sample t-tests were used to determine between group differences. This procedure is appropriate to test for differences among group means when the level of measurement for the independent variable (e.g., church attendance) is nominal and the level of measurement of the dependent variable (self-efficacy for exercise, religiosity, body image perception, income, education, BMI, age, and engagement in physical activity) is interval/ratio. The Chi-square test was used to assess proportional between group differences when the data did not meet the conditions of the two sample t-test.

\section{Specific Aim 3}

Explicate the predictors of self-efficacy for exercise and physical activity behavior among African American women.

Research Question 3A: What are the predictors of self-efficacy for exercise among African American women? 
Research Question 3B: What are the predictors of physical activity behavior among African American women?

Correlation and multiple regression analysis were used to determine the predictors of self-efficacy for exercise and routine engagement of physical activity. Prior to conducting regression analysis, categorical data were transformed into dummy codes where the presence of a characteristic was coded as 1 and the absence coded 0 . Additionally, effect coding was performed on categorical data with more than 2 categories (Munro, 2005). Correlation analyses were performed to determine the strength and association between predictor variables and the outcome variables. Independent variables were entered into the multiple regression models if the bivariate association between the predictor and outcome variables were significant at the .15 level.

\section{Consideration of Human Subjects}

The PI ensured consideration of human subjects through procedures such as obtaining written informed consent, identifying and minimizing risk to subjects, and maintaining confidentiality. A summary of the purposes of the study along with a summary of each questionnaire to be completed was included as the first document in the study packet. In addition to verbally verifying that all participants met the inclusion criteria of the study, each participant was asked their age prior to receiving a study packet to ensure that they qualified to participate. The PI verbally explained the consent document as participants read along and answered questions as needed. Participants were assured that participation was voluntary and that: (a) there were no right or wrong answers; (b) they could choose to exit the study at anytime; and (c) response to each was desired, but not required. Informed consent was considered implied for each participant who completed the questionnaires.

Potential risks to participants were minimal and related to completing the questionnaires that assessed demographic information, self-efficacy for exercise, religiosity, body image perception, and physical activity engagement. Minimal stress was a risk related to self disclosure of feelings and responding to questions about beliefs in God. The PI reiterated that there were no right or wrong answers on this form and that completion was strictly voluntary but that their input (response) was highly desired. Additionally, the heading on the GLHC instrument was modified to read "Locus of Health Control" rather than "God Locus of Health Control". Another potential risk was the time requirement for completing the study. The participants is this study were required to complete data requirements at specified times and locations.

Participants were provided with a monetary incentive and refreshments to offset the time associated with completing the study. The study was approved as an exempt by the University of Tennessee Health Science Center IRB to further ensure human subject protection.

This study provided opportunity for both direct and indirect personal and societal benefits. A potential indirect benefit of participating in this study was the satisfaction of 
knowing that the information provided by participants would be used to plan future exercise interventions tailored specifically for African American women. Additionally, participants could potentially experience the indirect benefit of increased self awareness related to physical activity behavior through completing the SBAS. Increased engagement in physical activity would facilitate a decreased rate of obesity and obesityrelated health complications. Thus, a potential societal benefit would be decreased financial burden on the healthcare system. The potential benefits of the study outweighed the minimal risk associated with this study.

Confidentiality was maintained throughout the study in several ways. All information collected for study purposes was confidential. Participants were not required to provide personal information such as names or date of birth. Each participant height and weight was recorded inside of the back cover of their numerically coded study packet. The PI obtained height and weight on individual participants in a private area during data collection sessions. Although no identifiable personal information was collected on participants all study packets were stored in a locked file cabinet in the PI's home. Access to data collected during this study was limited to the PI and the IRB approved research program advisor. While data collected during this study will be used in publications and presentations, confidentiality will not be breached because no identifiable information was collected. 


\section{CHAPTER 4. RESULTS}

This study had three related purposes. The investigator first explored the relationships among self-efficacy for exercise, religiosity, body image perception, body mass index, socioeconomic factors (i.e., level of education, monthly income, church attendance, residency [i.e., rural or urban], age, parental responsibility [i.e., caring for children under age 16], number of children, occupation and marital status), and physical activity behavior among African American women. Second, the investigator compared the level of self-efficacy for exercise, religiosity, body image perception, body mass index, socioeconomic factors (i.e., level of education, monthly income, church attendance, residency [i.e., rural or urban], age, parental responsibility [i.e., caring for children under age 16], number of children, occupation and marital status), and physical activity behavior among African American women among African American women that regularly attended church services (at least 3 times out of the month) to those who did not regularly attend church services (less than 3 times out of the month). Third, the investigator explicated the predictors of self-efficacy for exercise and physical activity behavior among African American women. The initial discussion in this chapter will provide an overview of the sample characteristics followed by corresponding statistical analysis for each research question (e.g., results).

\section{Sample Characteristics}

Sample characteristics represent means, standard deviations, frequencies, and percentages unless otherwise stated and are summarized in Table 1. The final sample consisted of 100 African American females. The mean age of the participants was 40 years. The mean monthly income was $\$ 2242$. On average participants attended church services 4 times a month, had completed high school or completed some undergraduate courses, were not married, and had 2 children. Most of the sample was obese $(67 \%)$, employed (75\%), resided within the city limits (74\%), and had at least one child under the age of $16(56 \%)$. Additionally, 61 participants (61\%) reported having occupations that required activity that approximated light intensity physical activity.

Table 2 depicts a summary of mean and median scores along with standard deviations for outcome variables for the entire sample. The PI did not identify established cut-points for self report measures use in this study hence mid-points on each scale was used for distinguishing categories.

Scores for the Physical Exercise Self-efficacy Scale (PESES), a measure of selfefficacy for exercise, ranged from 5 (lower self-efficacy for exercise) to 20 (higher selfefficacy for exercise) and indicated an individual's confidence in their exercise intentions. Total scores that ranged from 5 to 12 indicated less confidence in exercise intention and total scores that ranged from 13 to 20 indicated more confidence in exercise intention. 
Table 1. Demographic Characteristics of this Sample of African American Women ( $N$ $=100$ ).

\begin{tabular}{|c|c|c|}
\hline Characteristics & $\begin{array}{l}\text { Sample } \\
N(\%)\end{array}$ & $M \pm S D$ \\
\hline Age (years) & 100 & $40.0 \pm 11.0$ \\
\hline Monthly Income (in dollars) & 100 & $2242.0 \pm 1819.0$ \\
\hline Education (years) & 100 & $13.0 \pm 2.0$ \\
\hline Number of Children & 100 & $2.0 \pm 1.0$ \\
\hline Church Attendance (days/month) & 100 & $4.0 \pm 1.0$ \\
\hline BMI (calculated) & 100 & $34.0 \pm 8.0$ \\
\hline $\begin{array}{l}\text { Age Category } \\
\text { 21-34 (young adult) } \\
\text { 35-49 (middle-age adult) } \\
\text { 50-60 (older adult) }\end{array}$ & $\begin{array}{l}100 \\
48(48) \\
30(30) \\
22(22)\end{array}$ & \\
\hline $\begin{array}{l}\text { Income Category } \\
\quad \leq \$ 1699 \text { (lower) } \\
\quad \$ 1700-\$ 3299 \text { (middle) } \\
\quad \geq \$ 3300 \text { (upper) }\end{array}$ & $\begin{array}{l}100 \\
46(46) \\
33(33) \\
21(21)\end{array}$ & \\
\hline $\begin{array}{l}\text { Education Category } \\
\leq 12 \text { years (high school) } \\
\quad 13-16 \text { years (undergraduate) } \\
\geq 17 \text { years (graduate) }\end{array}$ & $\begin{array}{l}100 \\
48(48) \\
46(46) \\
6(6)\end{array}$ & \\
\hline $\begin{array}{l}\text { BMI Category } \\
\text { 18.5-24.9 Normal } \\
\text { 25.0-29.9 Overweight } \\
\geq 30.0 \text { Obese }\end{array}$ & $\begin{array}{l}100 \\
16(16) \\
17(17) \\
67(67)\end{array}$ & \\
\hline
\end{tabular}


Table 1. Cont'd.

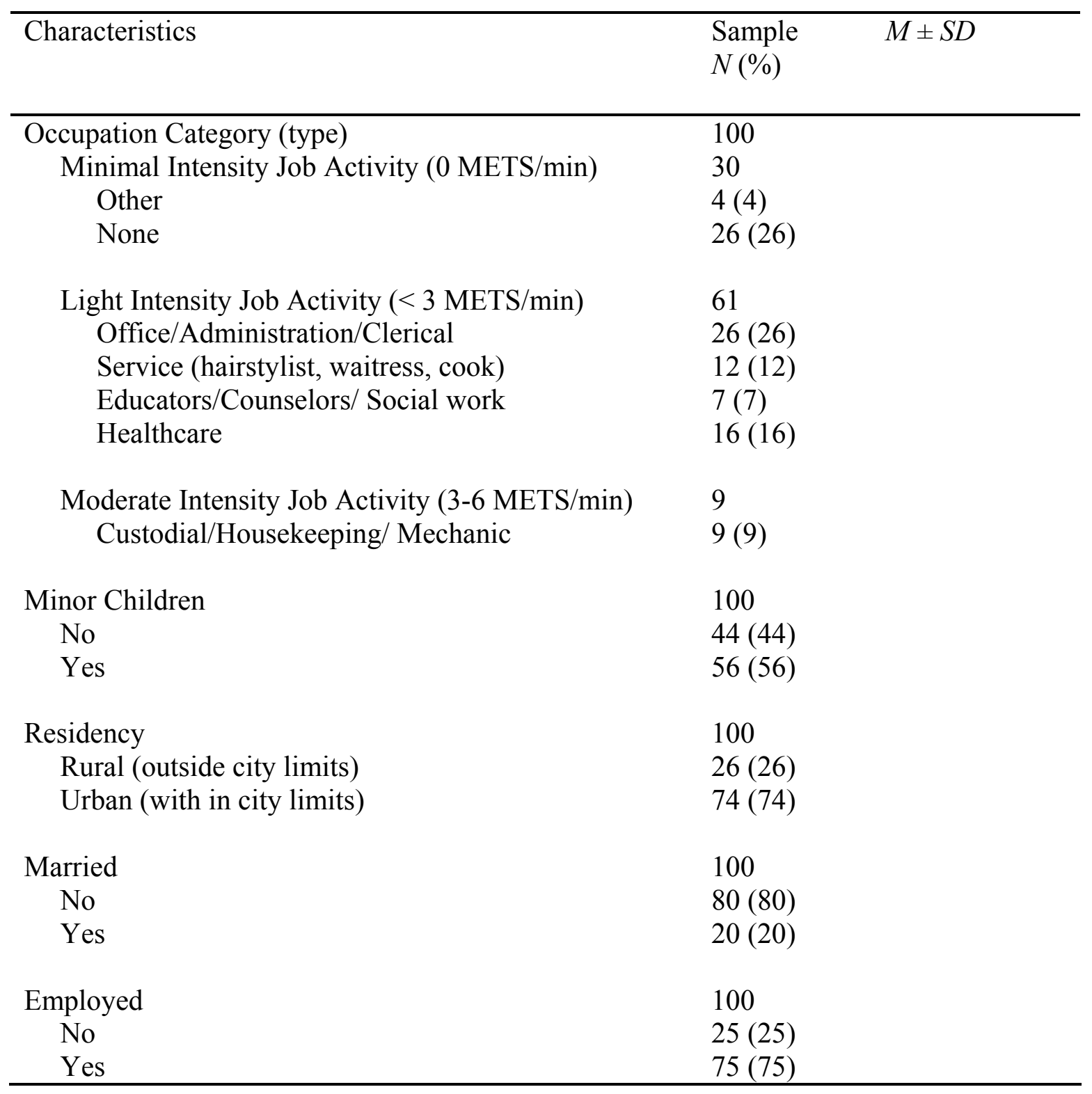


Table 2. Summarized Data for Physical Activity, Self-efficacy for Exercise, Religiosity, and Body Image Perception.

\begin{tabular}{|c|c|c|c|}
\hline Instrument & $\begin{array}{l}\text { Sample } \\
N(\%)\end{array}$ & $M \pm S D$ & Mdn \\
\hline $\begin{array}{l}\text { SBAS (total score) } \\
\text { SBAS (categories) } \\
\text { inactive } \\
\text { active }\end{array}$ & $\begin{array}{l}100 \\
78(78) \\
22(22)\end{array}$ & $2.2 \pm .88$ & 2.0 \\
\hline $\begin{array}{l}\text { PESES (total score) } \\
\text { PESES (categories) } \\
\text { lower } \\
\text { higher }\end{array}$ & $\begin{array}{l}100 \\
50(50) \\
50(50)\end{array}$ & $12.8 \pm 4.0$ & 12.5 \\
\hline $\begin{array}{c}\text { GLHC (total score) } \\
\text { GLHC (categories) } \\
\text { lower/intrinsic } \\
\text { higher/extrinsic }\end{array}$ & $\begin{array}{l}100 \\
33(33) \\
67(67)\end{array}$ & $20.4 \pm 9.4$ & 19.5 \\
\hline $\begin{array}{l}\text { ASI-R (total score) } \\
\text { ASI-R (categories) } \\
\text { poorer body image } \\
\text { better body image }\end{array}$ & $\begin{array}{l}100 \\
93(93) \\
7 \quad(7) \\
\end{array}$ & $3.4 \pm .55$ & 3.4 \\
\hline
\end{tabular}

SBAS $=$ Stanford Brief Physical Activity Scale, a measure of usual amount and intensity of physical activity.

PESES = Physical Exercise Self-efficacy Scale, a measure of self-efficacy for exercise $\mathrm{GLHC}=$ God Locus of Control Scale, a measure of religiosity.

ASI-R = Appearance Schema Inventory-Revised, a global measure of body image perception. 
Scores for the God Locus of Control Scale (GLHC), a measure of religiosity, ranged from 6 (low or intrinsic religiosity) to 36 (high or extrinsic religiosity) and indicate an individual's perception about religion. Total scores that ranged from 6 to 15 indicated an intrinsic religiosity that placed less responsibility for one's health on God and total scores that ranged from 16-36 indicated an extrinsic religiosity that placed more responsibility for one's health on God.

Scores for the Appearance Schema Inventory-Revised (ASI-R), a measure of body image perception, ranged from 1 (better perception) to 5 (poorer perception) and indicated an individual's perceptions about their own body image. Total scores that ranged from 1.0-2.5 indicated better body image perception and total scores that ranged from 2.6-5.0 indicated poorer body image perception.

Scores for the Stanford Brief Physical Activity Scale (SBAS), a measure of usual amount and intensity of physical activity, ranged from 1 (inactive) to 5 (hard intensity activity). Total scores that ranged from 1-2 on the SBAS indicated physical inactivity and total scores that ranged of 3-5 indicated being physically active. The BMI was calculated using individual height and weight and ranged from 19 (normal weight) to 53 (obese).

The following results reflect means plus or minus the standard deviation for the entire sample. The mean and standard deviation for self-efficacy for exercise was $12.80 \pm$ 4.03 for the entire sample. Fifty participants $(50 \%)$ were less confident in their exercise intentions and fifty participants $(50 \%)$ were more confident in their exercise intentions. The mean and standard deviation for GLHC was $20.4 \pm 9.35$ for the entire sample. Thirty-three participants (33\%) had more intrinsic religiosity and sixty-seven (67\%) participants had more extrinsic religiosity. The mean and standard deviation for the ASI-R was $3.37 \pm .55$ for the entire sample. Seven participants $(7 \%)$ had better body image perception and 93 participants $(93 \%)$ had poorer body image perception. The mean and standard deviation for the SBAS was $2.15 \pm .88$. Seventy-eight participants $(78 \%)$ did not engage in routine physical activity and 22 participants $(22 \%)$ did engage in routine physical activity. The mean BMI for the sample was $33.64 \pm 7.56$. Sixteen participants $(16 \%)$ were in the normal weight category, seventeen participants $(17 \%)$ were in the overweight, and sixty-seven participants (67\%) were in the obese category.

\section{Research Aims, Related Questions, and Findings}

Study findings are reported for each specific aim and question, respectively, with each aim and relevant research question restated followed by the results of the statistical analysis. Data analyses were conducted using the Statistical Package for the Social Science (SPSS) version 15.0. 


\section{Specific Aim 1}

Explore the relationships among self-efficacy for exercise, religiosity, body image perception, body mass index, socioeconomic factors (i.e., level of education, monthly income, church attendance, residency [i.e., rural or urban], age, parental responsibility [i.e., caring for children under age 16], number of children, occupation and marital status), and physical activity behavior among African American women.

\section{Question 1}

What are the relationships among self-efficacy for exercise, religiosity, body image perception, body mass index, socioeconomic factors (i.e., level of education, monthly income, church attendance, residency [i.e., rural or urban], age, parental responsibility [i.e., caring for children under age 16], number of children, occupation and marital status), and physical activity behavior among African American women?

\section{Findings}

Total scores for self-efficacy for exercise and religiosity were relatively normally distributed. Total scores for body image perception, BMI, level of education, monthly income, residency, number of children, routine engagement in physical activity and age were positively skewed while scores for church attendance, parental responsibility and marital status were negatively skewed. Prior to calculating correlation coefficients scatter plots were run to screen the data for evidence of linearity and for outliers in the dataset. Additionally, a normal log-transformation was performed on data with marked deviations from normality (i.e., skewness value $>-.4$ or $<.4$ ). Performing a normal logtransformation on skewed data improves distribution of the data and thus improves correlation coefficients (SPSS 15.0).

Using the bivariate correlation procedure, Pearson's product moment correlation coefficients were estimated for scale/interval data and Spearman's rho correlation coefficients were estimated on ordered data. A summary of the significant associations is included in Table 3. A weak negative association between religiosity (GLHC) and level of education $(\mathrm{r}=.28, \mathrm{p}=.00)$ was noted indicating that the more educated participants tended to place less responsibility for their health on God and vice-a-versa. A weak positive association between body image perception (ASI-R) and BMI $(r=.21, p=.03)$ was noted indicating that body image perception was worse among participants with higher BMI's increased. The weak positive association between BMI and level of education $(\mathrm{r}=.17, \mathrm{p}=.05)$ indicated that as the number of years of education increased so did BMI. There was a moderate positive association between level of education and monthly income $(\mathrm{r}=.48, \mathrm{p}=.00)$ indicating that as level of education increased monthly income increased and vice-a-versa. A weak positive association between monthly income and number of children $(\mathrm{r}=.20, \mathrm{p}=.05)$ was noted indicating that as monthly income increased number of children increased and vice-a-versa. A weak positive association between monthly income and church attendance $(\mathrm{r}=.23, \mathrm{p}=.02)$ was noted indicating 
Table 3. Summarized Data for Bivariate Correlation Analyses.

\begin{tabular}{llllllll}
\hline & GLHC & ASI-R & BMI & $\begin{array}{l}\text { Income } \\
\text { /Month }\end{array}$ & $\begin{array}{l}\# \\
\text { Children }\end{array}$ & SBAS & PESES \\
\hline Age & -.12 & -.07 & .12 & $.17^{*}$ & $.35^{* *}$ & .04 & .13 \\
\# Children & -.11 & -.14 & -.17 & $.20^{*}$ & 1 & .00 & .12 \\
Minor/ & -.05 & -.04 & -.08 & .10 & $.22^{*}$ & .03 & $-.21^{*}$ \\
Children & & & & & & & \\
SBAS & -.05 & -.03 & -.16 & $.24^{* *}$ & .00 & 1 & $.25^{*}$ \\
Education & $-.28^{* *}$ & .06 & $.17^{*}$ & $.48^{* *}$ & .04 & $.22^{*}$ & .06 \\
$\begin{array}{l}\text { Type } \\
\text { Occupation }\end{array}$ & .01 & .17 & .09 & $-.30^{* *}$ & .04 & $.32^{*}$ & -.09 \\
BMI & .05 & $.21 *$ & 1 & -.05 & -.17 & -.16 & $-.18^{*}$ \\
$\begin{array}{l}\text { Income/ } \\
\text { Month }\end{array}$ & -.14 & .08 & -.03 & 1 & .20 & .16 & .00 \\
$\begin{array}{l}\text { Church } \\
\text { Attendance }\end{array}$ & .04 & .06 & .09 & $.23^{*}$ & .07 & -.09 & $.20^{*}$ \\
Residence & -.10 & .01 & -.00 & -.07 & $.26^{*}$ & .01 & -.02 \\
\hline
\end{tabular}

GLHC $=$ God Locus of Control Scale

ASI-R = Appearance Schema Inventory-Revised Scale

$\mathrm{BMI}=$ body mass index

SBAS $=$ Stanford Brief Activity Survey

PESES = Physical Exercise Self-efficacy Scale

Sample size $=100 ; * \mathrm{p} \leq .05 ; * * \mathrm{p} \leq .01$. 
that as monthly income increased church attendance increased. A moderate positive association between age and number of children $(\mathrm{r}=.35, \mathrm{p}=.00)$ was noted indicating that older age was associated with having more children. The weak positive association between age and monthly income $(r=.17, \mathrm{p}=.05)$ indicated that older age was associated with higher income. Self-efficacy for exercise and church attendance was weakly and positively associated $(\mathrm{r}=.20, \mathrm{p}=.05)$, indicating that women who attended church frequently had higher confidence in their exercise intentions. There was a weak negative association between having minor children and self-efficacy for exercise $(r=-$ $.21, \mathrm{p}=.04)$ indicating that women with children under the age of 16 tended to have lower self-efficacy for exercise. The weak positive association between having minor children and residency $(\mathrm{r}=.26, \mathrm{p}=.01)$ indicated that women who had children under the age of 16 tended to reside within city limits. Self-efficacy for exercise and BMI were weakly and negatively associated $(\mathrm{r}=-.18, \mathrm{p}=.04)$ indicating that less confidence in exercise intentions was associated with higher BMI. A weak positive association between self-efficacy for exercise (PESES) and physical activity engagement (SBAS) $(r=.25, \mathrm{p}=$ $.01)$ was noted indicating that the more confident the participant was in their exercise intentions the more they engaged in routine physical activity and vice-a-versa.

Additionally, weak positive associations between physical activity engagement and education $(\mathrm{r}=.22, \mathrm{p}=.01)$ and income $(\mathrm{r}=.24, \mathrm{p}=.00)$ were noted. A moderate positive association was noted between physical activity engagement and type of occupation $(\mathrm{r}=$ $.32, \mathrm{p}=.00)$.

\section{Specific Aim 2}

Compare the level of self-efficacy for exercise, religiosity, body image perception, body mass index, socioeconomic factors (i.e., level of education, monthly income, church attendance, residency [i.e., rural or urban], age, parental responsibility [i.e., caring for children under age 16], number of children, occupation and marital status), and physical activity behavior among African American women that regularly attend church services (at least 3 times out of the month) to those who do not regularly attend church services (less than 3 times out of the month).

\section{Question 2}

What are the differences in self-efficacy for exercise, religiosity, body image perception, body mass index, socioeconomic factors (i.e., level of education, monthly income, church attendance, residency [i.e., rural or urban], age, parental responsibility [i.e., caring for children under age 16], number of children, occupation and marital status), and physical activity behavior among African American women that regularly attend church services (at least 3 times out of the month) to those who do not regularly attend church services (less than 3 times out of the month)? 


\section{$\underline{\text { Findings }}$}

Relevant variables were screened for normality. Total scores for physical activity, self-efficacy for exercise, and religiosity were slightly left skewed. Thus independent sample t test were chosen to estimate group means due to this test is robust to deviations from normality (Blalock, 1972). The Levene's test for equity of variance was performed (Glaser, 1983). Equal group variance was not present for all variables; hence results were report based on the significance of the Levene's test. Equal group variance is assumed if the significance value of the Levene's test exceeds .05 whereas unequal group variance is assumed if the significance value is less than .05. Additionally, the Chi-square test was used to assess proportional between group differences when the data did not meet the conditions of the sample t-test. The results of the Chi-square test are summarized in Table 4.

Analysis revealed that African American women that regularly attended church services (at least 3 times out of the month) and those who did not regularly attended church services reported similar levels of self-efficacy for exercise and routine engagement in physical activity. Additionally, African American women who lived in rural areas and those who resided within city limits reported similar levels of self-efficacy for exercise and routine engagement in physical activity. Twenty-six percent of the women in this study resided in rural areas. Fifteen percent of these rural participants reported middle to upper levels of income, 15\% reported completing high school or attending some college, $23 \%$ attended church frequently, $19 \%$ were obese, and $15 \%$ were middle-age or older adults. Chi-square analysis revealed a significant association between age category and church attendance $\left(X^{2}=6.17, \mathrm{p}=.05\right)$ Participants that attended church frequently reported higher scores on the GLHC scale (M $20.8 \pm S D$ 9.15), indicating more extrinsic religiosity compared to those who attended infrequently $(M 18.3 \pm S D$ 10.29), however the difference was insignificant $(\mathrm{t}=-.94, \mathrm{p}=.31)$. Likewise, participants residing in rural areas reported higher scores on the GLHC scale ( $M 22.0 \pm S D$ 7.9), indicating more extrinsic religiosity compared to those residing in urban areas $(M 19.84 \pm$ $S D$ 9.77); however the difference was insignificant $(\mathrm{t}=1.1, \mathrm{p}=.27)$. Among socioeconomic factors, a significant difference between mean age and church attendance $(\mathrm{t}=2.3, \mathrm{p}=.03)$ was noted. In this sample, African American women who attended church infrequently tended to be older $(M 44 \pm S D$ 12) than those who attended church frequently ( $M 37 \pm S D$ 10). Participants who attended church infrequently engaged in more physical activity $(M 2.3 \pm S D$ 1.26) than those who attended church frequently $(M$ $2.12 \pm S D$.78); however this difference was not significant $(\mathrm{t}=.17, \mathrm{p}=.87)$. Reported monthly income was slightly higher $(M 2377 \pm S D$ 1925) for those women who attended church frequently $(M 1579 \pm S D$ 952) compared to those who attended church infrequently; however this difference was marginally significant $(\mathrm{t}=-.19, \mathrm{p}=.06)$. Overall BMI was slightly higher $(M 34.3 \pm S D$ 6.94) for those women who attended church infrequently compared to those who attended church frequently ( $M 33.4 \pm S D$ 7.71); however this difference was not significant $(\mathrm{t}=.49, \mathrm{p}=.63)$. The average number of children for those participants who attended church frequently was slightly higher $(M$ $2.1 \pm S D$ 1.4) than for those who attended infrequently (M $2.2 \pm S D$ 1.2); however this difference was not significant $(\mathrm{t}=-.53, \mathrm{p}=-.60)$. 
Table 4. Chi-square Cross Tabulation of Demographic and Categorized Outcome Group Differences between African American Women That Attend Church Frequently and Those That Attend Infrequently.

\begin{tabular}{|c|c|c|c|}
\hline \multirow{2}{*}{\multicolumn{2}{|c|}{ Characteristic }} & \multirow{2}{*}{\multicolumn{2}{|c|}{ Church Attendance }} \\
\hline & & \multirow{2}{*}{$\begin{array}{c}\begin{array}{c}\text { Frequent } \\
n=83\end{array} \\
40\end{array}$} & \\
\hline Self-efficacy for & Lower & & $\begin{array}{c}\text { Infrequent } \\
n=17\end{array}$ \\
\hline Exercise & Higher & 43 & 7 \\
\hline \multirow[t]{2}{*}{ Religiosity } & Intrinsic & 25 & 8 \\
\hline & Extrinsic & 58 & 9 \\
\hline \multirow[t]{2}{*}{ Body Image } & Better & 6 & 1 \\
\hline & Poor & 77 & 16 \\
\hline Level of Physical & Active & 18 & 4 \\
\hline Activity & Inactive & 65 & 13 \\
\hline \multirow[t]{2}{*}{ Residency } & Rural & 23 & 3 \\
\hline & Urban & 60 & 14 \\
\hline \multirow[t]{3}{*}{ Income Category } & Lower & 36 & 10 \\
\hline & Middle & 27 & 6 \\
\hline & Upper & 20 & 1 \\
\hline \multirow[t]{3}{*}{ Education Category } & High School & 38 & 10 \\
\hline & Undergraduate & 40 & 6 \\
\hline & Graduate & 5 & 1 \\
\hline \multirow[t]{3}{*}{ BMI Category } & Normal & 15 & 1 \\
\hline & Overweight & 14 & 3 \\
\hline & Obese & 54 & 13 \\
\hline \multirow[t]{3}{*}{ Age Category* } & Young & 44 & 4 \\
\hline & Mid-age & 24 & 6 \\
\hline & Older & 15 & 7 \\
\hline \multirow[t]{2}{*}{ Married } & Yes & 17 & 3 \\
\hline & No & 66 & 14 \\
\hline \multirow[t]{2}{*}{ Minor Children } & Yes & 48 & 8 \\
\hline & No & 35 & 9 \\
\hline
\end{tabular}


Table 4. Cont'd

\begin{tabular}{cccc}
\hline \multirow{2}{*}{ Characteristic } & \multicolumn{2}{c}{ Church Attendance } \\
\cline { 3 - 4 } & & $\begin{array}{c}\text { Frequent } \\
n=83\end{array}$ & $\begin{array}{c}\text { Infrequent } \\
n=17\end{array}$ \\
& & & \\
\hline Type of Occupation & Minimal & 23 & 7 \\
& Light & 54 & 7 \\
& Moderate & 6 & 3 \\
\hline
\end{tabular}

$\mathrm{BMI}=$ body mass index

$* \mathrm{p} \leq .05$. Type of occupation categories represent on-the-job activity that is equivocal to MET's/min: Minimal = intensity that is sub-minimal MET's/min; Light = Intensity that is $<3 \mathrm{MET}$ 's/min; Moderate = Intensity that is between 3-6 MET's/min. 


\section{Specific Aim 3}

Explicate the predictors of self-efficacy for exercise and physical activity behavior among African American women.

\section{Question $3 A$} women?

What are the predictors of self-efficacy for exercise among African American

\section{Question $3 B$} women?

What are the predictors of physical activity behavior among African American

\section{$\underline{\text { Findings }}$}

Correlation and multiple regression analysis were used to determine the predictors of self-efficacy for exercise and routine engagement of physical activity. Before regression analyses were performed categorical data were transformed into dummy codes (Table 5), where the presence of a characteristic was coded as 1 and the absence coded 0 . Correlation analyses were performed to determine if there was a relationship between continuous independent variables (education, income, religiosity, self-efficacy for exercise, body image perception) and the outcome variables (self-efficacy for exercise and physical activity behavior).

The strength and significance of the linear relationship between the independent and dependant variables was used to determine which variables were entered into the regression analyses. Independent variables were entered into the multiple regression models if the bivariate association between the predictor and outcome variables were significant at the .15 level. Multicollinearity was not an issue in this study as all independent variables were weakly or moderately correlated. Multicollinearity becomes an issue in statistical analysis when independent variables are strongly correlated (Schroeder, 1990). Significant bivariate relationships are summarized in Table 3. Independent variables were entered or removed from the regression equation by the computer program based on the significance of the variable to explaining the variance in the outcome variable.

A series of separate multiple regression analyses using the stepwise and enter methods were conducted for each outcome variable (self-efficacy for exercise, physical activity behavior). Predicator variables included self-efficacy for exercise, BMI, monthly income, education, church attendance, occupation, age, married, level of physical activity, minor children, and number of children. Utilizing the enter method allowed for more explanation of the variance in self-efficacy for exercise and routine engagement in physical activity. The researcher must examine the $t$ statistic, in addition to the significance level of the independent variables in the model to determine its importance 
Table 5. Dummy Codes of Categorical String Variables.

\begin{tabular}{lc}
\hline Categorical Variables & Dummy Codes \\
\hline Married & 0 \\
No & 1 \\
Yes & \\
Residency & \\
$\quad$ Rural & 0 \\
$\quad$ Urban & 1 \\
SBAS & \\
Inactive & \\
$\quad$ Active & 0 \\
Minor Children & 1 \\
$\quad$ No & \\
$\quad$ Yes & \\
Church Attendance & 0 \\
Infrequent & 1 \\
Frequent & \\
\hline
\end{tabular}

SBAS $=$ Stanford Brief Activity Survey

$0=$ absence of characteristic

$1=$ presence of characteristic. 
in explaining the variance in the outcome variable. The following models display the results of the final regression analyses using the enter method. The first model shows the results (Table 6) of the independent variables (minor children, married, BMI, and level of physical activity) regressed upon self-efficacy for exercise. The independent variables in the model $(\mathrm{F}=3.56, \mathrm{p}<.05)$ explained $36 \%$ of the variance in self-efficacy for exercise. However, the independent variables minor children $(\mathrm{t}=-2.34, \mathrm{p}<.05)$, married $(\mathrm{t}=$ $-2.04, \mathrm{p}<.05)$, and BMI $(\mathrm{t}=2.19, \mathrm{p}<.05)$ were the most significant predictors in the model. Women that did not have children under the age of 16 , who were not married, and were in the normal weight BMI category had higher self-efficacy for exercise. The second model shows the results (Table 7) of the independent variables (occupation, selfefficacy for exercise, church attendance, BMI, monthly income) regressed upon engagement in physical activity. The independent variables in the model $(\mathrm{F}=3.27, \mathrm{p}<$ $.05)$ explained $17 \%$ of the variance in engagement in physical activity. However, the independent variable self-efficacy for exercise $(t=2.73, p<.05)$ was the best predictor of routine engagement in physical activity. 
Table 6. Summary of Multiple Regression Analysis for Self-efficacy for Exercise $(N$ $=100)$.

\begin{tabular}{lccc}
\hline Independent Variables & Beta & $\mathrm{t}$ & $p$ \\
\hline Minor children & -1.83 & -2.34 & .02 \\
Married & -1.97 & -2.04 & .02 \\
BMI category & 2.32 & 2.19 & .03 \\
Level of physical activity & -.12 & -.15 & .88 \\
\hline
\end{tabular}

$\mathrm{BMI}=$ body mass index

Note: Dependant Variable: Self-efficacy for Exercise; Model Statistics: $\mathrm{R}^{2}=.36, \mathrm{~F}=$ $3.56, \mathrm{df}=4,95, \mathrm{p}=.01$.

Table 7. Summary of Multiple Regression Analysis for Physical Activity Behavior $(N$ $=100)$.

\begin{tabular}{lccc}
\hline Independent Variables & Beta & $\mathrm{t}$ & $p$ \\
\hline Self-efficacy for Exercise & .27 & 2.73 & .01 \\
BMI calculated & -.15 & -1.54 & .13 \\
Income/month & .11 & 1.03 & .31 \\
Education/years & .11 & 1.04 & .30 \\
Church attendance & .17 & 1.69 & .09 \\
Type of occupation & .18 & 1.90 & .06 \\
\hline
\end{tabular}

$\mathrm{BMI}=$ body mass index

Note: Dependant Variable: Level of Physical Activity; Model Statistics: $\mathrm{R}^{2}=.17, \mathrm{~F}=$ $3.27, \mathrm{df}=6,93, \mathrm{p}=.01$. 


\section{CHAPTER 5. DISCUSSION AND IMPLICATIONS}

\section{Discussion}

This study investigated self-efficacy for exercise, religiosity, body image perception, BMI, socioeconomic factors, and physical activity behavior in African American women. Study findings also provided information related to factors that predicted self-efficacy for exercise and engagement in physical activity in this sample of African American women. This chapter includes a discussion of significant findings along with an appraisal of findings with respect to how they concur with or differ from published research studies. The discussion includes limitations and strengths of the study along with implications for theory, research, and practice.

\section{Demographic Findings}

The findings of this study were consistent with published literature with few noted exceptions. In this sample of African American women, 83\% reported attending church services at least 4 times per month. This finding is consistent with 2003 BRFSS data for the state of Alabama which reported $85 \%$ of African American women attended church services (Sanderson et al, 2003). Most of the sample (67\%) was categorized as obese, a finding that was consistent to current literature as well (Kim et al., 2006; Ogden et al., 2007; Wilcox et al., 2007; Yenek et al., 2001). Most women were single (80\%) and had children (86\%) consistent with other demographic findings in studies focusing on African American women (Chadiha et al., 2004; Dornelas et al., 2007; Sharma et al., 2005; Wallace-Williams et al., 2003). Additionally, $46 \%$ of the sample reported lower income levels consistent with other published findings focusing on African American women (Ball et al., 2007; Probst et al., 2004; Sanderson et al., 2003, Young et al., 2002). In contrast to other studies exploring these study variables, most (73\%) of this sample of African American women reported graduating from high school or attending college. This finding may be related to the fact that the current study was conducted in a Southern city that has several colleges and universities. Furthermore, the higher education level reported in this study sample when compared to other published studies could be evidence of socioeconomic disparity among African American women in different geographical area of the United States and should be further explored. According to the literature the socioeconomic status of most women in this study could present a barrier to physical activity engagement. Moreover, women in this study that reported caring for children under the age of sixteen experienced lower self-efficacy for exercise. The perceived or actual caregiver burden associated with being a single parent could lead to decreased self-efficacy for exercise and physical activity engagement (Henderson et al., 2000; Nies et al., 1999; Young et al., 2002). Most women in this study (80\%) were unmarried.

The obesity reported in this sample of African American women presents a threat to their affective stability and the excessive body weight could impact the amount and 
type of physical activity these women can engage in. According to social-cognitive theory efficacy beliefs are strengthened by mastery of task (Bandura, 1994). Thus it is imperative that physical activity interventions designed for African American women incorporate physical activities suitable for obese populations and gradually progress towards more intense or vigorous activities. Additionally, the prevalence of obesity in this sample of women further substantiates physical inactivity among this group.

Twenty-six percent of the women in this study resided in rural areas. Fifteen percent of these rural participants reported middle to upper levels of income, $15 \%$ reported completing high school or attending some college, $23 \%$ attended church frequently, 19\% were obese, and 15\% were middle-age or older adults. Although the number of rural dwelling participants $(n=26)$ was not comparable to the number of urban dwelling participants $(n=74)$, the aforementioned demographic findings support current literature, suggesting that high rates of obesity are prevalent among more educated African Americans that migrate to rural areas (Probst et al., 2004; Sallis et al., 1992; Scharff et al., 1999). The most plausible explanation for the disproportionate number of urban dwelling participants in this study is related to the convenience sampling and word of mouth recruitment methods employed. Most of the data collection sessions (3) were organized by individuals who previously completed the study. These participants recruited on behalf of the investigator and the recruitment sessions occurred in homes within the city limits. Moreover, the participants in these groups were often neighbors of the person recruiting individuals for the study. Additionally, the data collection sessions that were held in homes consisted of relatively large numbers of participants (10 to 20 per session). The findings of this study may not be a true reflection of the rural population.

Equal numbers of African American women in this study reported higher and lower self-efficacy for exercise; however the mean score reported on the PESES was $12.80 \pm 4.03$. Published findings indicated that African American women tend to have higher self-efficacy for exercise in general a finding that is consistent with the findings of this study (Felton et al., 2002; Sanderson et al., 2003). Although these women had relatively high confidence in their exercise intentions, $78 \%$ did not engage in routine physical activity. This finding is also consistent with published research (Felton et al., 2002; Sanderson et al., 2003). According to social-cognitive theory women in this study may be experiencing high confidence in exercise intentions based on past rather than current experience with physical activity engagement. Future health promotion intervention studies should be cognizant of this point because African American women may experience decreased self-efficacy for exercise upon reinitiating physical activity. Decreased self-efficacy for exercise could contribute to high attrition rates $(>40 \%)$ reported in other physical activity intervention studies. Additionally, some researchers have theorized that exercise behavior is not static but rather, dynamic (Barnett, Gauvin, Craig, \& Katzmarzyk, 2008; J. C. Graff, personal communication, August 1, 2008). This theory could explain the inconsistent study findings among groups in which physical activity behavior is measured as a fixed variable. 
The current study examined religiosity and church attendance with respect to physical activity among African American women. Most African American women in this study reported extrinsic religiosity (67\%). Several investigators have determined extrinsic religiosity by the frequency in which African American women participate in religious activities such as attending church services (Holt et al., 2005; Kinney et al., 2002; Koenig, et al., 1988). The findings of this study support current literature related to religiosity and church attendance; however there was no significant association between religiosity and physical activity behavior. Within this study sample the prevalence of frequent church attendance makes the African American church a feasible location for introducing physical activity interventions. Researchers will have to address the implications of the noted extrinsic religiosity among this group in order to strengthen interventions.

In contrast to some research findings and consistent with others, African American women in this study reported poorer body image perception if they had higher BMI's (Davis et al., 2005; Pumariega et al., 1994). The mixed results in the published literature are most likely related to discrepancies in measurement of this construct in African American women. The use of the multidimensional ASI-R scale to assess global body image perception among African American women likely provides a more accurate assessment of this construct in light of noted cultural variations. The association between increased BMI and poor body image in addition to the high prevalence of obesity noted among this sample of African American women suggests a potential affective barrier to physical activity engagement. Although the current study did not examine any variables in the affective component of social-cognitive theory, findings support further exploration of the effects of affective barriers such as depressive symptoms on selfefficacy for exercise and physical activity engagement.

\section{Relationships among Self-efficacy for Exercise, Religiosity, Body Image Perception, Body Mass Index, Socioeconomic Factors and Physical Activity Behavior}

The first research aim of this study focused on examining the relationships among self-efficacy for exercise, religiosity, body image perception, body mass index, socioeconomic factors (i.e., level of education, monthly income, church attendance, residency [i.e., rural or urban], age, parental responsibility [i.e., caring for children under age 16], number of children, occupation and marital status), and physical activity behavior in this sample of African American women. Several significant associations (Table 3) were noted.

Women in this study reported a more negative body image associated with increased BMI. Contrary to popular societal views, African American women in this group were displeased with being overweight or obese. The widely held cultural acceptance of increased body weight may not be salient in this sub-group of the African American population. There has been a historical trend towards improved body image perception among women without regard to race (Roberts et al., 2006). Additionally, differences in published studies examining body image perception among African 
American women may be attributed to measurement error. Current literature suggests that body image perception is multidimensional (Cash et al., 2002). Additionally, literature suggests that African American women hold a global view of beauty that includes various aspects such as style, spirit, and body (Flynn \& Fitzgibbon, 1998; R. W. Johnson \& Broadnax, 2003; Ruben et al., 2003). Hence, it is more appropriate to consider both appearance and motivational aspects of body image when assessing global perceptions in African American women. The findings of this study reflect global body image among this sample of African American women; however explicating the predictors of body image perception among this group would be beneficial in planning future culturallysensitive health promotion interventions.

Many significant socioeconomic associations were found in this study as well. A higher level of monthly income was associated with older age, having more education, having more children, engaging in more physical activity, and attending church more frequently. Moreover, older women in the study tended to have more children; however those children tended to be older than 16 years of age. This finding suggest that decreased parental responsibilities associated with having non-dependant children allows older women more time to engage in physical activity.

African American women with lower levels of education placed more responsibility for their health on God, had a lower BMI, and engaged in less physical activity. Women that had minor children reported lower self-efficacy for exercise and rural residency. Women with higher self-efficacy for exercise attended church services more frequently and engaged in more routine physical activity. Additionally, women that had low intensity physical activity occupations such as clerical or teaching positions engaged in less routine physical activity.

The aforementioned socioeconomic associations contribute to overall selfefficacy for exercise according to the social-cognitive theory (Bandura, 1994; Gecas, 1989). Interventions designed to increase self-efficacy for exercise as a means of promoting physical activity should focus on the socioeconomic aspects found in the selection component rather than the motivational component of the social-cognitive theory. According to the social-cognitive theory, published literature, and the findings of this study lower education and income level is associated with less engagement in physical activity (Ball et al., 2007; Bandura, 1994; Clark \& Nothwehr, 1999; Richther et al., 2002; Sanderson et al., 2003; Wise, et al., 2006). Additionally, addressing barriers to physical activity such as providing child care could prove to be very beneficial to this group. The availability of such a resource could also decrease perceived stress thus positively contributing to self-efficacy for exercise. Although, women with lower education levels had lower BMI's it is important to note that most of the participants in this study were overweight or obese. The affective implications of having poorer body image that was associated with higher BMI in this study could impact overall selfefficacy for exercise and engagement in physical activity. Additionally, the prevalence of low intensity occupation and its association to physical inactivity in this study could represent perceptions that equate "going to work" with physical activity engagement. 
Further exploration of this association would be beneficial to understanding persistent physical inactivity within this group.

\section{Group Differences: Frequent Church Attendance and Infrequent Church Attendance}

The second aim of this study was to examine the differences in the level of selfefficacy for exercise, religiosity, body image perception, body mass index, socioeconomic factors (i.e., level of education, monthly income, church attendance, residency [i.e., rural or urban], age, parental responsibility [i.e., caring for children under age 16], number of children, occupation and marital status), and physical activity behavior among African American women that attend church services frequently compared to those who attend infrequently. Participants that attended church frequently as well as those who resided in rural areas, reported slightly higher scores on the GLHC indicating more extrinsic religiosity compared to those who attended infrequently. Although African American women that attended church services frequently reported similar levels of self-efficacy for exercise and routine engagement in physical activity compared to those who attended infrequently, there were noted differences among socioeconomic factors. In this study African American women that attended church frequently were younger, less physically active, had more children, had slightly higher income, and had slightly lower BMI's compared to those that attended church service infrequently. Individuals may perceive barriers to physical activity differently based on things such as personal circumstance, perceptions, and access. Hence, further examination of specific modifiable barriers to physical activity could be beneficial in explaining physical activity behavior among sub-groups within the African American female population. Consider the following scenario with respect to potential barriers to engaging in physical activity: a young African American woman, who is a single parent

of minor children, currently working part-time and attending school to become a nurse. If the findings of this study and other research are sound then the barriers faced in the proposed example would likely change with time, children get older, graduating, increased earnings, etc. In this instance younger African American women could be more vulnerable to physical inactivity. The notion that engagement in physical activity is cyclic further supports the need for consideration of age differences among African American women. There is a dearth of research examining the differences among African American women who attend church frequently compared to those who attend infrequently. Although there is adequate documentation of the overall prevalence of church attendance among African American women, this setting alone may not be sufficient for reaching those members of the group that are most vulnerable to physical inactivity.

\section{Predictors of Self-efficacy for Exercise and Physical Activity Behavior}

The third and final aim of this study was to explicate the predictors of selfefficacy for exercise and physical activity behavior among African American women. 
Several studies have examined the correlates of self-efficacy for exercise among African American women (Breitkopf et al., 2007; Felton et al., 2002; Hawkins et al., 2006; Henderson et al., 2000; Miller et al., 1998; Young et al., 2002). This study sought to determine which socioeconomic factors best predicted self-efficacy for exercise. The predictor model that best explained the variance in self-efficacy for exercise included the following independent variables: BMI, marital status, education, parental responsibility, occupation, and engagement in physical activity. The most significant predictors of selfefficacy for exercise were minor children, married, and BMI category. In this sample, women that had children under the age of 16 years old, that were married, and that were overweight or obese had less confidence in the exercise intentions. The predictor model that best explained the variance in physical activity behavior included: type of occupation, self-efficacy for exercise, church attendance, BMI, monthly income. However, the most significant predictor of physical activity behavior was self-efficacy for exercise. In this sample women that were physically active were more confident in their exercise intentions. These findings are quite meaningful for understanding persistent physical inactivity among African American women. Studies consistently show that African American women do not engage in adequate amounts of physical activity in spite of their noted confidence in their exercise intentions (Dornelas et al., 2007; Kim et al., 2006; Kumanyika \& Charleston, 1992; McNabb et al., 1997; Wilcox et al., 2007; Yenek et al., 2001; Young \& Stewart, 2006). Consistent with current research most of the participants in this study were confident in their exercise intentions and physically inactivity.

Prior research using social-cognitive theory has focused on motivational aspects of the theory by including African Americans as key study personnel, encouraging workout companions, and tapping into the social networks within the church setting (Kim et al., 2006; Yenek et al., 2001). Researchers might find it more beneficial to focus on selections aspects of the social-cognitive theory by focusing on relevant modifiable barriers to physical activity in this group as a means of promoting physical activity. Education on what constitutes moderate intensity physical activity could counter possible perception of "going to work" as engagement in physical activity and foster a clearer perception of level of physical activity. Based on the findings of this study and other research, assisting African American women to overcome barriers related to parental responsibility could facilitate more engagement in routine physical activity which in turn would increase self-efficacy. Theoretically this increased self-efficacy could lead to sustained engagement in routine physical activity.

\section{Limitations and Strengths}

This study had several limitations and strengths. Limitations of the study were related to sampling strategies and instrumentation. Strengths of the study include expounding on socioeconomic factors that influence self-efficacy for exercise and examination of physical activity behavior among African American women. Limitations are discussed first followed by a discussion of the strengths of this study. 
Most of the participants in this study resided in urban areas of the southern United States, thus it is inappropriate to generalize the results to African American women living in other geographical regions or to those with differing demographic characteristics.

African American women in this study were primarily obese, well educated, and single, earned a lower-middle income, had children, and were employed. While these demographic characteristics likely influence physical activity behavior to varying degrees, health status is an important factor that was not assessed. Information related to health status was not obtained in this study, thus producing a threat to the generalizability and internal validity.

The descriptive correlational study design included a non-probability sample. Convenience sampling limits the external validity of the study findings. Additionally the unanticipated snowball sampling that occurred yielded a large number of frequent church attendees, thus placing the study at risk for sampling bias which presents a threat to the internal and external validity of the study. The use of self-assessment tools may not truly reflect the true attitudes or experiences of these women; however the goal of the study was to assess their perceptions for most of the study dependent and independent variables.

Despite the limitations, there were noted strengths associated with this study. The fact that many investigators have recognized and used social-cognitive theory to guide their research in African American female populations speaks to the usefulness of this theory to address phenomena that may be complicated by complex issues such as culture. Use of this theory in intervention studies that promote physical activity however has been relatively unsuccessful in producing the desired increase in physical activity levels needed to create health benefit such as a decreased prevalence of obesity. These studies have focused primarily on the motivational components of social-cognitive theory to promote engagement in physical activity. Using African American aerobic instructors and peer work-out companions have been cited as sources on motivation in past intervention studies (Kim et al., 2006; Yenek et al., 2001). According to social-cognitive theory, motivation based on goals such as increased engagement in physical activity is primarily mediated by internal influences; this internally influenced motivation may explain the ineffectiveness of prior intervention studies in achieving their physical activity outcomes. The current study is strengthened by the fact that barriers such as parental responsibility, residency, level of education, and level of income of physical activity were examined within the context of social-cognitive theory. This approach allowed for the illumination of relevant barriers within the selection component of the social-cognitive theory that affect self-efficacy for exercise in African American women. The barriers identified in this component of social-cognitive theory are strongly affected by individual choices, thus making them modifiable. Overcoming these modifiable barriers could plausibly facilitate sustained engagement in routine physical activity behavior among African American women. 


\section{Implication: Theory, Research, and Practice}

The findings of this study present several implications for theory, research, and health promotion practice. Many models and theories have been used in health promotion practice; however the social-cognitive theory often serves as the bases for these theories (Kim et al., 2006; Wilcox et al., 2007; Yenek et al., 2001). Often these modified theories do not comprehensively address complex cultural issues and their relevance to cultural sub-groups. Appropriate use of the social-cognitive theory allows for identification of specific factors that affect task or behaviors such as engagement in physical activity. Researchers should recognize and focus on the modifiable factors that mediate selfefficacy which in turn affects behavior. Theoretically, identification of modifiable socioeconomic factors, such as childcare issues that are perceived barriers to physical activity among African American women would facilitate implementation of culturallysensitive strategies to address these factors. The increased perceived familial responsibility observed in African American women is often attributed to culture. Although it may not be feasible to change the perception noted above, providing childcare would be a feasible culturally-sensitive research approach to address this factor and facilitate participation.

Members of the African American population have a unique culture with many variations that are not fully understood by the research community. These variations likely affect every aspect of life for members of this population and subgroups within this population. Additionally variations within sub-groups make it difficult to generalize population based research findings. Such seems to be the case with African American women, a sub-group within the African American population. Much of what is known about African American culture is derived from aggregate based research and may not be appropriate for discovering the underlying cause of persistent physical inactivity among women in this population. Religiosity is understood to be a central theme within African American culture, a point that is supported by the findings of this study and other research (Chatters et al., 1992; K. S. Johnson et al., 2005; Milevsky \& Levitt, 2004). However the findings of this study suggest that this belief does not directly affect exercise behavior. Research has suggested that members of the African American population experience less body image disturbance with increased BMI (Altabe, 1998; Breitkopf \& Littleton, 2007; Cash et al., 2002; Celio et al., 2002; Miller et al., 2000; Roberts et al., 2006; Thomas et al., 2008). Many of the studies reporting this perception among African American women have been qualitative rather than quantitative and may reflect the recognition of the collective cultural belief but may not reflect individual perceptions of body image. The quantitative findings of this study suggest that the above is a misconception as the women in this study experienced poorer body image perception with higher BMI.

Recent faith-based health promotion studies have been implemented through the African American church, in part because of the noted importance of religion among this group (Kim et al., 2006; Wilcox et al., 2007; Yenek et al., 2001). This health promotion practice has allowed for a captive group of participants, however high attrition rates have been problematic. Researchers should recognize that African American women attending 
church frequently may not be representative of the entire group. African American women in this study that reported frequent church attendance tended to be older women. Moreover older women in this study engaged in routine physical activity more than younger women. Furthermore simply placing these interventions in the church doesn't fully address the cultural importance of religion to many members of this ethnic group. This study found that those women who attended church more frequently also reported an extrinsic religiosity, which was representative of an attitude that placed more responsibility for one's health on God. This knowledge could be beneficial in understanding the high attrition rates in past church and faith-based intervention studies. This perception could cause a decreased overall appraisal of routine physical activity. Researchers may find it beneficial to incorporate scripture into various aspects of the intervention in addition to conducting interventions in the church. According to the social-cognitive theory this approach would be culturally-sensitive to this group's choice to frequently attend church; however recognizing this culture's perceptions related to religion through the inclusion of scripture (God's written word) could serve as a source of external motivation. Theoretically, this approach could counter attrition caused by decreased appraisal for physical activity because participation would be based on appraisal of religion not physical activity.

\section{Summary and Recommendations for Further Research}

Persistent physical inactivity is a complex phenomenon. Cultural variations within African American female populations related to barriers to physical activity, socioeconomic disparities, and body image perception have been noted in this study. Religiosity was not directly associated with physical activity behavior in this study. However, there was a significant positive association between religiosity and educational attainment. Level of education was among the socioeconomic factors the indirectly (significantly positively associated with income) influenced self-efficacy for exercise and physical activity behavior. Women that chose to attend church frequently and those that resided in rural areas reported more extrinsic religiosity. Additionally, women across all age groups in this study that attended church frequently engaged in less routine physical activity. Self-efficacy for exercise was positively associated with physical activity and church attendance. Further exploration of the influence of religiosity and church attendance on self-efficacy for exercise and physical activity behavior is recommended.

The majority of the women in this study reported being rather confident in their exercise intentions however they were physically inactive. Regression analysis in this study explained $36 \%$ and $17 \%$ of the variance in self-efficacy for exercise and physical activity behavior, respectively. Although the outcome variables were explained in this study, the percentage of explained variance suggests the need to explore additional socioeconomic factors within this group. Younger women with lower income in this study attended church less frequently. As could be expected younger women in this study were caring for minor children more often than older women. The parental responsibility of caring for minor children was a noted barrier to engagement in physical activity and predicted self-efficacy for exercise in this sample of African American women. 
Additionally, the majority of women in this study were not married. In this sample being married was associated with lower self-efficacy for exercise. In-depth exploration of influence of being partnered, either married or cohabitating and physical activity behavior of those partners is recommended to further illuminate physical activity behavior among African American women.

Past research of physical activity behavior in the African American female population has often employed a positivist view toward understanding this complex phenomenon. The findings of this study support the use of the social-cognitive theory as a guide to research in the African American female population. Through the use of this theory, researchers can apply the findings from past research in a more holistic manner to fully understand physical activity behavior in this group. Furthermore, the findings of this study suggest that health promotion intervention implemented through the church could potentially exclude a vulnerable portion of this group. Future research should focus on further examination of modifiable socioeconomic factors that influence self-efficacy for exercise and physical activity behavior among African American women.

Focus group discussions with African American women that attend church frequently and infrequently should be conducted separately to identify sub-group variations. Researchers might find great benefit from conducting focus group interviews with African American women in different age categories to identify relevant modifiable barriers. The information obtained could then be used in conjunction with a CBPR approach to tailor intervention to meet the needs of those women that attend church frequently and for those that attend infrequently. Future health promotion interventions might find great benefit by addressing modifiable environmental barriers in conjunction with the African American church. Interventions that place open access structures such as playgrounds surrounded by walking tracts on church grounds would recognize this culture's religious perceptions and socioeconomic barriers related to unaffordable access. This strategy could allow for inexpensive or free access to all African American women regardless of their attendance habits. Additionally this strategy would promote engagement in physical activity by introducing an actual and perceived safe environment for engaging in physical activity in the African American community. This approach would also address child care to a certain extent as well. According to the socialcognitive theory and other published research self-efficacy for exercise is enhanced by seeing others engage in physical activity. In the context of the social-cognitive theory, implications of such an intervention are far reaching and could potentially impact the entire family and African American population. Furthermore, the implications of increased engagement in physical activity among African Americans include decreased prevalence of obesity, improved health outcomes, and decreased financial healthcare burden.

In conclusion, the findings of this study illuminated the significant positive association between self-efficacy for exercise and physical activity behavior in African American women. Information related to the predictors of self-efficacy for exercise and physical activity behavior will assist researchers plan more tailored culturally relevant health promotion interventions for this population. 


\section{LIST OF REFERENCES}

Ai, A. L., Peterson, C., Rodgers, W., \& Tice, T. N. (2005). Effects of faith and secular factors on locus of control in middle-aged and older cardiac patients. Aging \& Mental Health, 9(5), 470-481.

Allport, G. W., \& Ross, J. M. (1967). Personal religion orientation and prejudice. Journal of Personality and Social Psychology, 5, 432-443.

Altabe, M. (1998). Ethnicity and body image: Quantitative and qualitative analysis. International Journal of Eating Disorders, 23, 153-159.

Annesi, J. J., \& Whitaker, A. C. (2008). Relations of mood and exercise with weight loss in formerly sedentary obese women. American Journal of Health Behavior, 32(6), 676-683.

Ard, J. D., \& Rosati, R., et al. (2000). Culturally-sensitive weight loss program produces significant reduction in weight, blood pressure, and cholesterol in eight weeks. Journal of National Medical Association, 92(11), 515-523.

Ark, P. D., Hull, P. C., Husaini, B. A., \& Craun, C. (2006). Religiosity, religious coping styles, and health service use: Racial differences among elderly women. Journal of Gerontological Nursing, 32(8), 20-29.

Atchley, R. C. (1997). The subjective importance of being religious and its effect on health and morale 14 years later. Journal of Aging Studies, 11, 131-141.

Ball, K., Timperio, A., Salmon, J., Giles-Corti, B., Roberts, R., \& Crawford, D. (2007). Personal, social and environmental determinates of educational inequalities in walking: A multilevel study. Journal of Epidemiology Community Health, 61, 108-114.

Bandura, A. (1994). Self-efficacy. In Ramachaudran, V. S. (Ed.), Encyclopedia of human behavior (Vol. 4, pp. 71-81). New York: Academic Press.

Bandura, A. (1997). Self-efficacy: The exercise of control. New York: Freeman.

Banks-Wallace, J., \& Parks, L. (2004). It's all sacred: African American women's perspectives on spirituality. Issues in Mental Health Nursing, 25, 25-45.

Barnett, T. A., Gauvin, L., Craig, C. L., \& Katzmarzyk, P. T. (2008). Distinct trajectories of leisure time physical activity and predictors of trajectory class membership: A 22 year cohort study. International Journal of Behavioral Nutrition and Physical Activity, 5(1), 57-64. 
Bettencourt, B. A., Talley, A., Benjamin, A. J., \& Valentine, J. (2006). Personality and aggressive behavior under provoking and neutral conditions: A meta-analytic review. Psychological Bulletin, 132(5), 751-777.

Black, H. (1999). Poverty and prayer: Spirituality narratives of older African American women. Review of Religious Research, 40(4), 359-374.

Blalock, H. M. (1972). Social statistics. New York: McGraw-Hill.

Breitkopf, C. R., \& Littleton, A. B. (2007). Body image: A study in a tri-ethnic sample of low income women. Sex Roles, 56, 373-380.

Brown, S. A. (2005). Measuring perceived benefits and perceived barriers for physical activity. American Journal Health Behaviors, 29(2), 107-116.

Cash, T. F., Fleming, E. C., Alindogan, J., Steadman, L., \& Whitehead, A. (2002). Beyond body image as a trait: The development and validation of the Body Image States Scale. Eating Disorders, 10, 103-113.

Cash, T. F., Melnyk, S. E., \& Hrabosky, J. I. (2004). The assessment of body image investment: An extensive revision of the Appearance Schema Inventory. International Journal of Eating Disorders, 35, 305-316.

Celio, A. A., Zabinski, M. F., \& Wilfley, D. E. (2002). African American body images. In Cash, T. F., Pruzinsky, T (Ed.), Body image: A handbook of theory, research, and clinical practice. New York: Guilford Press.

Centers for Disease Control and Prevention. (2002). Overwieght and obesity Retrieved January 15, 2007, from http://www.cdc.gov/nccdphp/dnpa/obesity

Centers for Disease Control and Prevention. (2009). Healthy weight: It's not a diet, it's a lifestyle! Retrieved January 20, 2009, from http://www.cdc.gov/healthyweight/assessing/bmi/adult_bmi/index.html

Chadiha, L. A., Adams, P., Biegel, W. A., \& Gutierrez, L. (2004). Empowering African American women informal caregivers: A literature synthesis and practice strategies. Social Work, 49(1), 97-108.

Chatters, L. M., Levin, J. S., \& Taylor, R. J. (1992). Antecedents and dimensions of religious involvement among older black adults. Journal of Gerontology: Social Science, 47, S269-S278.

Clark, D. O., \& Nothwehr, F. (1999). Exercise self-efficacy and its correlates among socioeconomically disadvantaged older adults. Health Education \& Behavior, 26, 535-546. 
Cohen, J. (1988). Multiple regression and correlation analysis. In Statistical power analysis for the behavioral science (2nd ed., pp. 407-465). Hillsdale, New Jersey: Lawrence Erlbaum Associates.

Craft, L., L., Freund, K., M., Cupepper, L., \& Perna, F. M. (2007). Intervention study of exercise for depressive symptoms in women. Journal of Women's Health, 16(10), 1499-1509.

Culture. (n.d.). The American heritage new dictionary of cultural literacy (3 ed.). Boston: Houghton Mifflin Company.

Davis, E. M., Clark, J. M., Carrese, J. A., Gary, T. L., \& Cooper, L. A. (2005). Racial and socioeconomic differences in the weight-loss experiences of obese women. American Journal of Public Health, 95(9), 1539-1543.

Dornelas, E. A., Stepnowski, R. R., Fischer, E. H., \& Thompson, P. D. (2007). Urban ethnic minority women's attendance at health clinic vs. church-based exercise programs. Journal of Cross Cultural Gerontology, 22, 129-136.

Ester, M. F., Mireille, N. M., Jos, W. R., \& Twisk, W. V. (2006). Physical activity measurements affect participants' behavior in a randomized controlled trial. Clinical Epidemiology, 59, 404-411.

Fallon, E. A., Wilcox, S., \& Ainsworth, B. E. (2005). Correlates of self-efficacy for physical activity in African American women. Women \& Health, 41(3), 47-62.

Felton, G. M., Boyd, M. D., Bartoces, M., \& Tavakoli, A. S. (2002). Physical activity in young African American women. Health Care for Women International, 23, $905-$ 918.

Fleury, J., Harrell, J. S., \& Cobb, B. (2001). Regular physical activity in older African Americans. In Funk, S., Tournquist, E., Leeman, J., Miles, M. \& Harrell, J. (Eds.), Key aspects of preventing and managing chronic illness (pp. 85-94). New York: Springer.

Flynn, K. J., \& Fitzgibbon, M. (1998). Body images and obesity risk among black females: A review of the literature. Annals of Behavioral Medicine, 20(1), 13-24.

Franzoni, F., \& Ghiadoni, L., et al. (2005). Physical activity plasma antioxidant capacity and endothelium-dependent vasodilation in young and older men. American Journal of Hypertension, 18 (4 part 1), 510-600.

Gecas, V. (1989). The social psychology of self-efficacy. Annual Review of Sociology, 15, 291-316.

Glaser, R. E. (1983). Levene's robust test of homogeneity of variance, Encyclopedia of statistical sciences 4 (pp. 608-610). New York: Wiley. 
Godfrey-Smith, P. (2003). Theory and reality: An introduction to the philosophy of science (1 ed.). Chicago: University of Chicago.

Green, B. L., Haldeman, G. F., Kaminski, K. N., Neal, K., Lim, S. S., \& Conn, D. L. (2006). Factors affecting physical activity behavior in urban adults with arthritis who are predominantly African American and female. Physical Therapy, 86(4), 510-519.

Grote, N. K., Bledsoe, S. E., Larkin, J., Lemay, E. P., \& Brown, C. (2007). Stress exposure and depression in disadvantaged women: The protective effects of optimism and perceived control. Social Worker Research, 31(1), 19-32.

Hawkins, B., Tuff, R. A., \& Dudley, G. (2006). African American women, body composition, and physical activity. Journal of African American Studies, 10(1), 44-56.

Henderson, K. A., \& Ainsworth, B. E. (2000). Sociocultural perspectives on physical activity in the lives of older African American and American Indian women: A cross cultural activity participation study. Women \& Health, 31(1).

Holt, C. L., Lewellyn, L. A., \& Rathweg, M. J. (2005). Exploring religion-health mediators among African American parishioners. Journal of Health Psychology, 10(4), 511-527.

Jacobson, T. A., Morton, F., Jacobson, K. L., Sharma, S., \& Garcia, D. C. (2002). An assessment of obesity among African American women in an inner city primary care clinic. Journal of the National Medical Association, 94, 1049-1057.

Johnson, K. S., Elbert-Avila, K. I., \& Tulsky, J. A. (2005). The influence of spiritual beliefs and practices on the treatment preferences of African Americans: A review of the literature. Journal of the American Geriatrics Society, 53(4), 711-719.

Johnson, R. W., \& Broadnax, P. A. (2003). A perspective on obesity. The ABNF Journal, 69-70.

Jones, P. (1992). African American women: The psychotherapeutic process as a coping style, American Psychological Association conference. Washington, DC.

Kim, K. H., Linnan, L., Campbell, M. K., Brooks, C., Koenig, H. G., \& Wiesen, C. (2006). The WORD (Wholeness, Oneness, Righteousness, Deliverance): A faithbased weight-loss program utilizing a community-based participatory research approach. Health Education \& Behavior, XX(X), 1-17.

Kinney, A. Y., Emery, G., Dudley, W. N., \& Croyle, R. T. (2002). Screening behaviors among African American women at high risk for breast cancer: Do beliefs about God matter. $O N F, 29(5), 835-843$. 
Koenig, H. G., Moberg, D. O., \& Kval, J. N. (1988). Religious activities and attitudes of older adults in a geriatric assessment clinic. Journal of the American Geriatric Society, 36, 362-374.

Kumanyika, S. K., \& Charleston, J. B. (1992). Lose weight and win: A church-based weight loss program for blood pressure control among black women. Patient Education Counsel, 19(1), 19-32.

Matthews, H. F., Lannin, D. R., \& Mitchell, J. P. (1994). Black women's narratives from eastern North Carolina. Soc Sci Med, 38, 789-800.

McNabb, W., Quinn, M., Kerver, J., Cook, S., \& Karrison, T. (1997). The PATHWAYS church-based weight loss program for urban African American women at risk for diabetes. Diabetes Care, 20(10), 1518-1523.

Milevsky, A., \& Levitt, M. J. (2004). Intrinsic and extrinsic religiosity in preadolescence and adolescense: Effect on psychological adjustment. Mental Health, Religion, \& Culture, 4, 307-321.

Miller, K. J., Gleaves, D. H., Hirsch, T. G., Green, B. A., Snow, A. C., \& Chanda, C. C. (2000). Comparison of body image dimensions by race/ethnicity and gender in a university population. International Journal of Eating Disorders, 27, 310-316.

Moshki, M., Ghofranipour, F., Hajizadeh, E., \& Azadfallah, P. (2007). Validity and reliability of the Multidimensional Health Locus of Control Scale for college students. BMC Public Health, 7, 295-300.

Munro, B. H. (2005). Statistical methods for health care research (5th ed.). Philadelphia: Lippincott Williams \& Wilkins.

Murray, T. S., Goggin, K., \& Malcarne, V. L. (2006). Development and validation of the Alcohol-Related God Locus of Control Scale. Addictive Behaviors, 31, 553-558.

National Institute of Health. (2009). Obesity education initiative: Guide to physical activity. Retrieved March 7, 2009, from http://www.nhlbi.nih.gov/health/public/heart/obesity/lose wt/phy act.htm

National Institute of Mental Health. (2009). What are the signs and symptoms of depression? Retrieved August 1, 2009, from http://www.nimh.nih.gov/health/publications/depression/what-are-the-signs-andsymptoms-of-depression.shtml

Nies, M. A., Vollman, M., \& Cook, T. (1999). African American women's experiences with physical activity in their daily lives. Public Health Nursing, 16(1), 23-31.

Ogden, C. L., Carroll, M. D., McDowell, M. A., \& Flegal, K. M. (2007). Obesity among adults in the United States: No statistically significant change since 2003-2004. Hyattsville, MD: National Center for Health Statistics. 
Pargament, K. I. (1997). The psychology of religion and coping. New York: Guildford Press.

Payne, I. R., Bergin, A. E., Bielema, K., A., \& Jenkins, P. H. (1991). Review of religion and mental health: Prevention and the enhancement of psychosocial functioning. Prevention in Human Services, 9, 11-40.

Plantinga, A. J., \& Bernell, S. (2004). A spatial economic analysis of urban land use and obesity Journal of Regional Science, 45(3), 473-495.

Polit, D. F., \& Beck, C. T. (2004). Nursing research: Principles and methods (7 ed.). Philadelphia: Lippincott Williams and Wilkins.

Powell, L. M., Slater, S., Chaloupka, F. J., \& Harper, D. (2006). Availability of physical activity-related facilities and neighborhood demographics and sociodemographic characteristics: A national study. American Journal of Public Health, 96(9), 16761680.

Probst, J. C., Moore, C. G., Glover, S. H., \& Samuels, M. E. (2004). Person and place: The compounding effects of race/ethnicity and rurality on health. American Journal of Public Health, 94(10), 1695-1703.

Pumariega, A. J., Gustavson, C. R., Gustavson, J. C., Motes, P. S., \& Ayers, S. (1994). Eating attitudes in African American women: The essence eating disorder survey. Eating Disorders: The Journal of Treatment and Prevention, 2, 5-16.

Resnicow, K., Baranowski, T., Ahluwalia, J. S., \& Braithwaite, R. L. (1999). Cultural sensitivity in public health: Defined and demystified. Ethnicity \& Disease, 9(1), $10-21$.

Resnicow, K., Jackson, A., Braithwaite, C. D., Blisset, D., Rahotep, S., \& Periasamy, S. (2002). Healthy body/healthy spirit: A church-based nutrition and physical activity intervention. Health Education Research, Theory and Practice, 17(5), $562-573$.

Richter, D. L., Wilcox, S., Greaney, M. L., Henderson, K. A., \& Ainsworth, B. E. (2002). Environmental, policy, and cultural factors related to physical activity in African American women. Women \& Health, 36(2), 91-109.

Roberts, A., Feingold, A., Cash, T. F., \& Johnson, B. T. (2006). Are black-white differences in females' body dissatisfaction decreasing? A meta-analytic review. Journal of Consulting and Clinical Psychology, 74(6), 1121-1131.

Robinson, B. K., \& Wicks, M. N. (2007). Religiosity, exercise self-efficacy, and African American women: A pilot study. 
Rubin, L. R., Fitts, M. L., \& Becker, A. E. (2003). "Whatever feels good in my soul": Body ethics and aesthetics among African American and Latina women. Culture, Medicine and Psychiatry, 27, 49-75.

Sallis, J. F., Hovell, M. F., \& Hofstetter, C. R. (1992). Predictors of adaption and maintenance of vigorous physical activity in men and women. Prev Med, 21, 237251.

Sanderson, B. K., Foushee, H. R., Bittner, V., Cornell, C. E., Stalker, V., \& Shelton, S., et al (2003). Personal, social, and physical environmental correlates of physical activity in rural African American women in Alabama. American Journal of Preventive Medicine, 25(3Si), 30-37.

Scharff, D. P., Homan, S., Kreuter, M., \& Brennan, L. (1999). Factors associated with physical activity in women across the life span: Implications for program development. Women \& Health, 29, 115-134.

Schroeder, M. (1990). Diagnosing and dealing with multicollinearity. Western Journal of Nursing Research, 12(2), 175-187.

Schwarzer, R., \& Renner, B. (2000). Social-cognitive predictors of health behavior: Action self-efficacy and coping self-efficacy. Health Psychology, 19, 487-495.

Schwarzer, R., \& Renner, B. (ND). Health-specific self-efficacy scales. Retrieved March 20, 2007, from http://userpage.fu-berlin.de/ health/healself.pdf

Sharma, M., Sargent, L., \& Stacy, R. (2005). Predictors of leisure-time physical activity among African American women. American Journal of Health Behavior, 29(4), 352-359.

Sniehotta, F. F., Scholz, U., \& Schwarzer, R. (2005). Bridging the intention-behavior gap: Planning, self-efficacy, action control in the adoption and maintenance of physical exercise. Psychology and Health, 20(2), 143-160.

Staffileno, A. M., Coke, L. A., \& Hollenberg, S. M. (2007). Blood pressure responses to lifestyle physical activity among young, hypertension-prone, African American women. Journal of Cardiovascular Nursing, 22(2), 107-111.

Taylor-Piliae, R. E., Norton, L. C., Haskell, W. L., Mahbouda, M. H., Fair, J. M., \& Iribarren, C., et al. (2006). Validation of a new brief physical activity survey among men and women aged 60-69 years. American Journal of Epidemiology, 164(6), 598-606.

Thomas, A. M., Moseley, G., Stallings, R., Nichols-English, G., \& Wagner, P. J. (2008). Perceptions of obesity: Black and white differences. Journal of Cultural Diversity, 15(4), 174-180. 
Trust for America's Health. (2008). F is for fat: How obesity policies are failing in America. Retrieved January 15, 2007, from http://www.healthyamericans.org

U.S. Census Bureau. (2000). Census 2000 urban and rural classification. Retrieved February 1, 2009, from http://www.census.gov/geo/www/ua/ua_2k.html

U.S. Department of Health and Human Services. Healthy people 2010. Retrieved January 15, 2007, from http://www.healthypeople.gov/LHI/lhiwhat.htm

U.S. Department of Health and Human Services. (1996). Physical activity and health: A report from the Surgeon General. Atlanta, Ga.

Wallace-Williams, S., Dilworth-Anderson, P., \& Goodwin, P. Y. (2003). Caregiver role strain: The contribution of multiple roles and available resources in AfricanAmerican women. Aging \& Mental Health, 7(2), 103-112.

Wallston, K. A., Malcarne, V. L., Flores, L., Hansdottir, I., Smith, C. A., \& Stein, M. J., et al. (1999). Does God determine your health? The God Locus of Health Control Scale. Cognitive Therapy and Research, 23(2), 131-142.

Wilbur, J., Miller, A. M., Chandler, P., \& McDevitt, J. (2003). Determinants of physical activity and adherence to a 24 -week home-based walking program in African America and Caucasian women. Research in Nursing and Health, 26, 213-229.

Wilcox, S., Laken, M., Bopp, M., Gethers, O., Huang, P., \& McClorin, L. (2007). Increasing physical activity among church members: Community-based participatory research. American Journal of Preventive Medicine, 32(2), 131-138.

Wilson-Ford, V. (1992). Health protective behaviors of rural black elderly women. Health Social Work, 17, 28-36.

Wise, L. A., Adams-Campbell, L. L., Palmer, J. R., \& Rosenberg, L. (2006). Leisure time physical activity in relation to depressive symptoms in the Black Women's Health study. Annals of Behavioral Medicine, 32(1), 68-76.

Wolfe, W. A. (2000). Obesity and the African-American woman: A cultural tolerance of fatness or other neglected factors. Ethnic Disparity, 10(3), 446-453.

World Health Organization. (1948). WHO definition of health. Retrieved July 1, 2007, from http://www.who.int/about/definition/en/print.ht

Yenek, L. R., Becker, D. M., Moy, T. F., Gittelsohn, J., \& Koffman, D. M. (2001). Project joy: Faith-based cardiovascular health promotion for African American women. Public Health Report, 116(1), 68-81. 
Young, D. R., He, X., Genkinger, J., Sapun, M., Mabry, I., \& Jehn, M. (2004). Health status among urban African American women: Associations among well-being, perceived stress, and demographic factors. Journal of Behavioral Medicine, 27(1), 63-76.

Young, D. R., He, X., Harris, J., \& Mabry, I. (2002). Environmental, policy, and cultural factors related to physical activity in well-educated urban African American women. Women \& Health, 36(2), 29-41.

Young, D. R., \& Stewart, K. J. (2006). A church-based physical activity intervention for African American women. Family and Community Health, 29(2), 103-117. 
APPENDICES 


\section{Appendix A. Written Permission/Proof of Purchase for Study Instruments}

\section{Body Images Research Consulting}

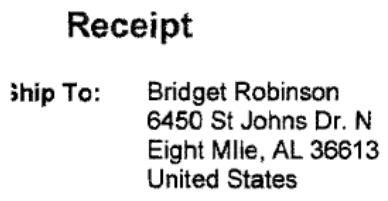

Receipt

Ship To: Bridget Robinson 6450 St Johns Dr. N

Eight Mlie, AL 36613

United States

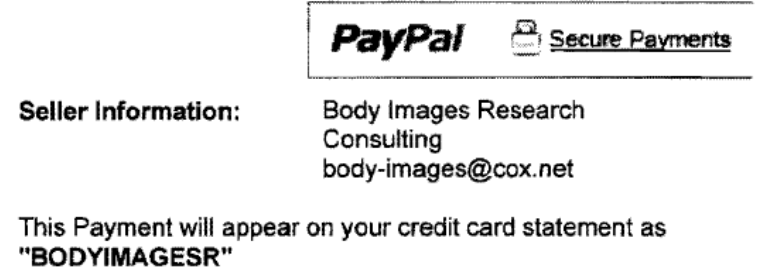

"BODYIMAGESR"

Receipt ID: 2479-3350-8687-3823

Placed on Oct. 2, 2008

\begin{tabular}{|c|c|c|c|}
\hline Item & Options & Quantity & Amount \\
\hline \multirow[t]{3}{*}{$\begin{array}{l}\text { Appearance Schemas inventory-Revised (ASI-R) } \\
\text { Item \# ASI-R }\end{array}$} & & 1 & $\$ 20.00$ \\
\hline & & Subtotal: & $\$ 20.00$ USD \\
\hline & & Total Amount: & $\$ 20.00$ USD \\
\hline
\end{tabular}


>>> Ruth Taylor-Piliae <rpiliae@stanford.edu> 08/22/07 7:38 PM $\gg>>$ 22 August 2007

Dear Ms. Robinson,

Congratulations on your pursuit of higher education! Thank you for your interest in my research on physical activity and use of the Stanford Brief Activity Survey (SBAS). First of all you have our permission to use the SBAS for your dissertation.

The original tool validation of the SBAS was conducted in the healthy older controls, comparing the SBAS to a 7-day physical activity recall interviewer-administered questionnaire, and then we also examined the associations between SBAS with selected CVD risk factors.

Taylor-Piliae, R.E., Norton, L.C., Haskell, W.L., Mahbouba, M.H., Fair, J.M., Iribarren, C., Hlatky, M.A., Go, A.S., \& Fortmann, S.P. Validation of a New Brief Physical Activity Survey Among Men and Women Age 60-69 Years. American Joumal of Epidemiology, 164(6): 598-606, 2006.

A 2 nd validation study was then done in the younger cases with $C A D$ to see if we could replicate the findings, e.g, the associations between selected CVD disease risk factors across the SBAS activity levels, by gender.

Taylor-Piliae, R.E., Haskell, W.L., Iribarren, C., Norton, L.C. Mahbouba, M., Fair, J.M., Hlatky, M.A., Go, A.S., \& Fortmann, S.P. Clinical Utility of the Stanford Brief Activity Survey in Men and Women with Early-Onset Coronary Artery Disease. Journal of Cardiopulmonary Rehabilitation and Prevention 27(4): 227-232, 2007.

The study was representative of the race/ethnicity groups in the San Francisco Bay area. So we did have African American women in that study. I would be happy to speak with you further about use of the SBAS tool in your research study, or answer additional question that you might have. 


\section{Appendix B. Standardized Demographic Data Form}

Almost done.... The following information, along with the other questionnaires, will help in the future planning of faith-base programs and intervention for our African American community. I really appreciate your completing the remaining questions and for participating in my study.

Are you African American/Black? YES NO

Are you employed?

YES NO

If you answered "Yes" to question \# 2 what is your occupation ; Skip this question if you answered "No"

Are you female?

YES NO

What is your age?

What is your average household monthly income?

What is the highest grade you completed in school?

How many children do you have? ; What are their ages?

Please list any support persons (i.e., husband, mother, friend, etc.) that you have.

How often do you attend church services each month (i.e., once a month; every Sunday, etc.) 


\section{Appendix C. Physical Exercise Self-Efficacy Scale}

For each statement below, please fill in the bubble above the appropriate response. Please make sure that you answer EVERY ITEM and that you mark ONLY ONE bubble per statement. This is a measure of your personal belief, thus there are no right or wrong answers.

\begin{abstract}
I can manage to carry out my exercise intentions even when I have worries and problems.

I can manage to carry out my exercise intentions even if I feel depressed.
\end{abstract}

I can manage to carry out my exercise intentions even when I feel tense.

I can manage to carry out my exercise intentions even when I'm tired.

I can manage to carry out my exercise intentions even when I am busy.

$\mathrm{O}$

very uncertain

$\mathrm{O}$

very uncertain

$\mathrm{O}$

very uncertain

$\mathrm{O}$

very uncertain

$\mathrm{O}$

very uncertain

$\mathrm{O}$

rather uncertain

$\mathrm{O}$

rather uncertain

$\mathrm{O}$

rather uncertain

$\mathrm{O}$

rather uncertain

$\mathrm{O}$

rather uncertain

\section{$\mathrm{O}$}

rather certain

$\mathrm{O}$

rather certain

$\mathrm{O}$

rather certain

O

rather certain

$\mathrm{O}$

rather certain
$\mathrm{O}$

very certain

$\mathrm{O}$

very certain

$\mathrm{O}$

very certain

$\mathrm{O}$

very certain

$\mathrm{O}$

very certain 


\section{Appendix D. God Locus of Health Control Scale}

Instructions: Each item below is a belief statement about your health with which you may agree or disagree. Beside each statement is a scale which ranges from strongly disagree (1) to strongly agree (6). For each item I would like you to circle the number that represents the extent to which you agree or disagree with the statement. The more you agree with the statement, the higher will be the number you circle. The more you disagree with a statement, the lower will be the number you circle. Please make sure you answer EVERY ITEM and that you circle ONLY ONE number per item. This is a measure of your personal beliefs, thus there are no right or wrong answers.

\begin{tabular}{|l|l|}
\hline $\begin{array}{l}\text { 1= Strongly Disagree (SD) } \\
\text { 2= Moderately Disagree (MD) }\end{array}$ & $\begin{array}{l}4=\text { Slightly Agree (A) } \\
5=\text { Moderately Agree (MA) } \\
\text { 3= Slightly Disagree (D) }\end{array}$ \\
$6=$ Strongly Agree (SA) \\
\hline
\end{tabular}

If my health worsens, it is up to God to determine whether I feel better SD MD again.

Most things that affect my health happen because of God.

God is directly responsible for my health getting better or worse

Whatever happens to my health is God's will

Whether or not my health improves is up to God.

God is in control of my health.

$\begin{array}{cccccc}\text { SD } & \text { MD } & \text { D } & \text { A } & \text { MA } & \text { SA } \\ 1 & 2 & 3 & 4 & 5 & 6 \\ 1 & 2 & 3 & 4 & 5 & 6 \\ 1 & 2 & 3 & 4 & 5 & 6 \\ 1 & 2 & 3 & 4 & 5 & 6 \\ 1 & 2 & 3 & 4 & 5 & 6 \\ 1 & 2 & 3 & 4 & 5 & 6\end{array}$




\title{
Appendix E. Stanford Brief Activity Survey
}

\author{
Stanford Brief Activity Survey ${ }^{1}$
}

Instructions

This is a self-administered questionnaire that attempts to have the respondent summarize his/her usual physical activity on-thejob and during leisure-time during the past year, based on a single statement for each. Section I pertains to on-the-job activity, while Section II pertains to leisure-time activity.

Give the questionnaire to the participant and ask him/her to read through the entire questionnaire before answering. Remind them that they should select the answer that best represents their activity during the past year. They need to provide an answer for both their on-the-job and leisure-time activity. If they are not gainfully employed outside the home but work regularly around the house, they should include this activity in the on-the-job section.

'Taylor-Piliae, R E., Norton, L.C., Haskell, W L., Mahbouda, MH., Fair, J.M., Iribarren, C., Hlatky, MA., Go, A. S., \& Fortmann, S.P. Validation of a New Brief Physical Activity Survey Among Men and Women Age 60-69 Years. American Jownal of Epidomiology, 164 (๑): 598-606, 2006. 


\section{Instrument}

Section I: On-The-Job Activity

Please check the box next to the one statement that best describes the kinds of physical activity you usually performed while on the job this last year. If you are not gainfully employed outside the home but perform work around the home regularly, indicate that activity in this section.

\begin{tabular}{|c|l|}
\hline A. O & If you have no job or regular work, check Box A and go on to Section II. \\
\hline B. O & $\begin{array}{l}\text { I spent most of the day sitting or standing. When I was at work I did such things as writing, typing, talking on the } \\
\text { telephone, assembling small parts or operating a machine that takes very little exertion or strength. If I drove a car or } \\
\text { truck while at work, I did not lift or carry anything for more that a few minutes each day. }\end{array}$ \\
\hline C. O & $\begin{array}{l}\text { I spent most of the day walking or using my hands and arms in work that required moderate exertion. When I was at } \\
\text { work I did such things as delivering mail, patrolling on guard duty, mechanical work on automobiles or other large } \\
\text { machines, house painting or operating a machine that requires some moderate activity work of me. If I drove a truck } \\
\text { or lift, my job required me to lift and carry things frequently. }\end{array}$ \\
\hline D. O & $\begin{array}{l}\text { I spent most of the day lifting or carrying heavy objects or moving most of my body in some other way. When I was } \\
\text { at work, I did such things as stacking cargo or inventory, handling parts or materials, or I did work like that of a } \\
\text { carpenter who builds structures or a gardener who does most of the work without machines. }\end{array}$ \\
\hline E. O & $\begin{array}{l}\text { I spent most of the day doing hard physical labor. When I was at work I did such things as digging or chopping with } \\
\text { heavy tools, or carrying heavy loads (bricks, for example) to the place where they are to be used. If I drove a truck or } \\
\text { operated equipment, my job also required me to do hard physical work most of the day with only short breaks. }\end{array}$ \\
\hline
\end{tabular}

Section II: Leisure-time Activity

Please check the box next to the one statement which best describes the way you spent your leisure-time during most of the last year.

\begin{tabular}{|c|l|}
\hline F. O & $\begin{array}{l}\text { Most of my leisure time was spent without very much physical activity. I mostly did things like watching television, } \\
\text { reading or playing cards. If I did anything else, it was likely to be light chores around the house or yard, or some } \\
\text { easy-going game like bowling or catch. Only occasionally, no more than once or twice a month, did I do anything } \\
\text { more vigorous, like jogging, playing tennis or active gardening. }\end{array}$ \\
\hline G. O & $\begin{array}{l}\text { Weekdays, when I got home from work, I did few active things. But most weekends I was able to get outdoors for } \\
\text { some light exercise- going for walks, playing a round of golf (without motorized carts), or doing some active chores } \\
\text { around the house. }\end{array}$ \\
\hline H. O & $\begin{array}{l}\text { Three times per week, on the average, I engaged in some moderate activity- such as brisk walking or slow jogging, } \\
\text { swimming or riding a bilke for 15-20 minutes or more. Or I spent 45 minutes to an hour or more doing moderately } \\
\text { difficult chores- such as raking or washing windows, mowing the lawn or vacuuming, or playing games such a } \\
\text { doubles tennis or basketball. }\end{array}$ \\
\hline I. O & $\begin{array}{l}\text { During my leisure time over the past year, I engaged in a regular program of physical fitness involving some kind of } \\
\text { heavy physical activity at least three times per week. Examples of heavy physical activity are: jogging, running or } \\
\text { riding fast on a bicycle for 30 minutes or more; heavy gardening or other chores for an hour or more; active games or } \\
\text { sports such as handball or tennis for an hour or more; or a regular program involving calisthenics and jogging or the } \\
\text { equivalent for } 30 \text { minute or more. }\end{array}$ \\
\hline J. O & $\begin{array}{l}\text { Over the past year I engaged in a regular program of physical fitness along the lines described in the last paragraph } \\
\text { (I), but I did it almost daily- five or more times per week. }\end{array}$ \\
\hline
\end{tabular}




\section{Scoring}

Using the color-coded scoring table, determine the intersection of the respondent's on-the-job activity (A-E) response on the vertical axis, with their leisure-time activity (F-J) response on the horizontal axis, to indicate their current activity category. Each color represents a different activity category, as follows: Red=Inactive, Yellow=Light-intensity Activity, Green=Moderate-Intensity Activity, Blue=Hard-Intensity Activity, Lavender=Very Hard-Intensity Activity.

\begin{tabular}{|c|c|c|c|c|c|c|}
\hline & \multicolumn{6}{|c|}{ Leisure-time activity (F-J) } \\
\hline \multirow{6}{*}{$\begin{array}{c}\text { On-the-Job } \\
\text { Activity } \\
\text { (A-E) }\end{array}$} & & $\mathbf{F}$ & G & $\mathrm{H}$ & I & $\mathrm{J}$ \\
\hline & $A$ & & & & & \\
\hline & B & & & & & \\
\hline & $\mathrm{C}$ & & & & & \\
\hline & D & & & & & \\
\hline & 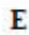 & & & & & \\
\hline
\end{tabular}

Examples

Example \#1: a person that works in an office mainly sitting at a desk all day, and rides a bicycle for 30 minutes/day, $5 \mathrm{x} /$ week would choose " $\mathrm{B}$ " as their on-the-job activity and " $\mathrm{J}$ " as their leisure-time activity. The intersection of these two responses on the colorcoded table would place them in a very-hard intensity activity category (lavender color)

Example \#2: a person who works full-time as a carpenter performing some hard labor during the day, and plays golf once a week on the weekends; would choose " $D$ " as their on-the-job activity and "G" as their leisure-time activity. The intersection of these two responses on the color-coded table would place them in a moderate-intensity activity category (green color). 


\section{Appendix F. Appearance Schema Inventory-Revised}

\section{The Beliefs about Appearance Questionnaire (ASI-R Short Form)}

The statements below are beliefs that people may or may not have about their physical appearance and its influence on life. Decide on the extent to which you personally disagree or agree with each statement and enter a number from 1 to 5 in the space on the left. There are no right or wrong answers. Just be truthful about your personal beliefs.

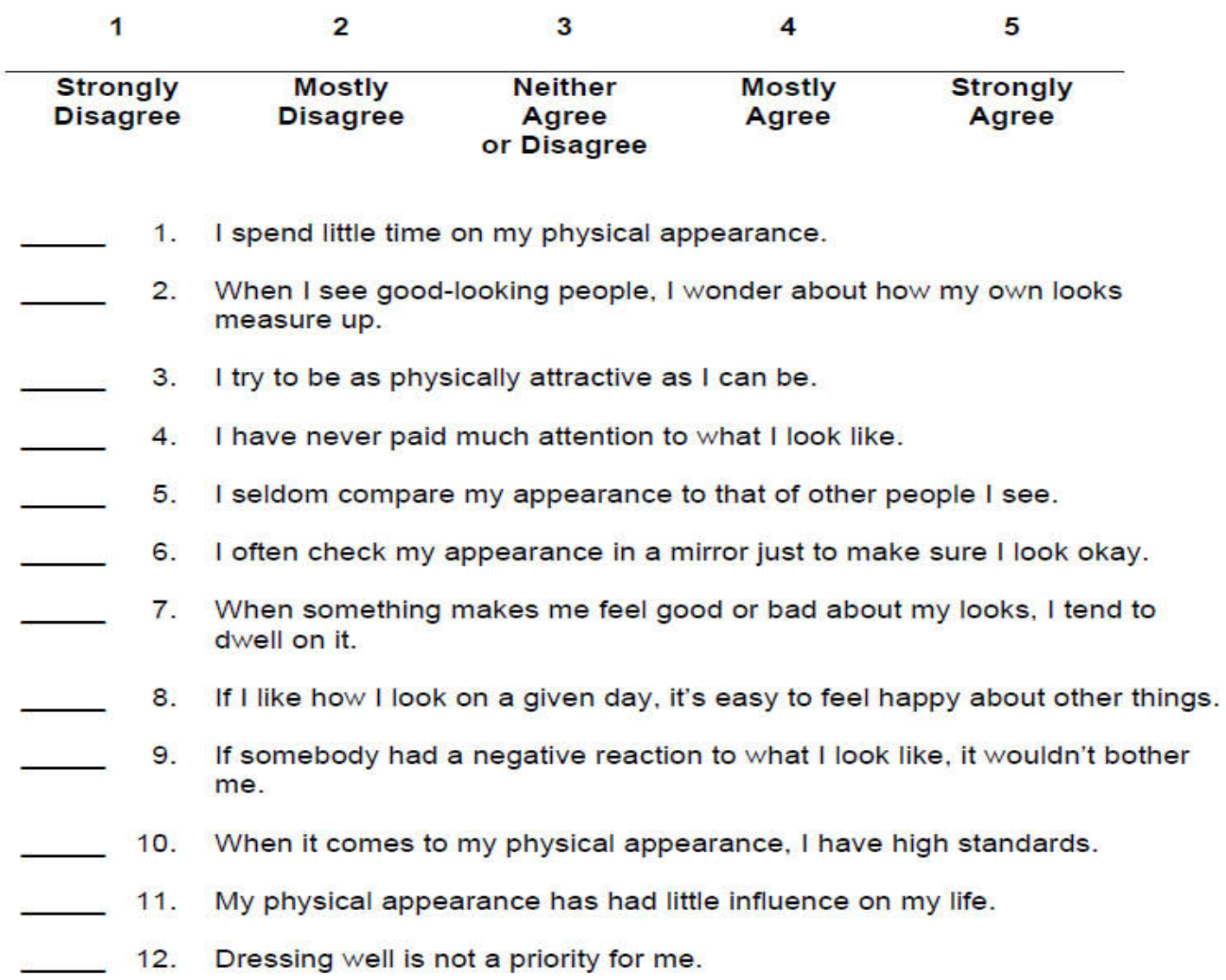

(continued on the next page) 


\section{Strongly Disagree}

Mostly
Disagree

Neither
Agree
or Disagree

Mostly

Agree

Strongly or Disagree

Agree

13. When I meet people for the first time, I wonder what they think about how I look.

14. In my everyday life, lots of things happen that make me think about what I look like.

15. If I dislike how I look on a given day, it's hard to feel happy about other things.

16. I fantasize about what it would be like to be better looking than I am.

17. Before going out, I make sure that I look as good as I possibly can.

18. What I look like is an important part of who I am.

19. By controlling my appearance, I can control many of the social and emotional events in my life.

20. My appearance is responsible for much of what's happened to me in my life.

(ASI-R ๑Thomas F. Cash, Ph.D., 2003) 


\section{Appendix G. Institutional Review Board Approval Letter}

\begin{tabular}{lr}
\hline THE UNIVERSITY OF TENNESSEE \\
Health Science Center \\
\hline \\
Institutional Review Board \\
910 Madison Avenue, Suite 600 \\
Memphis, TN 38163 \\
Tel: (901) 448-4824
\end{tabular}

December 17, 2008

Bridget Katina Robinson

College of Nursing

Department of Nursing

Re: IRB\# 08-00192-XM: Study Title: Self Efficacy for Exercise, Religiosity, BMI and Body Image Perception: Determinates of Physical activity behavior among African American Women of Faith

Dear Ms. Robinson,

The Administrative Section of the UTHSC Institutional Review Board (IRB) reviewed your application for the above referenced project.

The Administrative Section of the IRB determined your application to be consistent with the guidelines $f$ exempt review under 45CFR46.101(b)(2). In accord with 45CFR46.116 (d), informed consent may 1 altered, with the cover statement used in lieu of an informed consent interview. The requirement to secure signed consent form is waived under 45CFR46.117(c) (2). Willingness of the subject to participate wi constitute adequate documentation of consent. Therefore your application has been determined to comp with proper consideration for the rights and welfare of human subjects and the regulatory requirements for $\mathrm{tl}$ protection of human subjects. This letter constitutes full approval of your application, questionnaires, da collection tools and the consent cover statement, stamped approved by the IRB on December 17, 2008 for tl above referenced study.

In the event that volunteers are to be recruited using solicitation materials, such as brochures, posters, wel based advertisements, etc., these materials must receive prior approval of the IRB.

Any alterations (revisions) in the protocol, questionnaires or consent cover statement must be prompt submitted to and approved by the UTHSC Institutional Review Board prior to implementation of the: revisions. You have individual responsibility for reporting to the Board in the event of unanticipated , serious adverse events and subject deaths.

Sincerely,

Signature applied by Terrence F Ackerman on 12/19/2008 10:16:14 AM CST

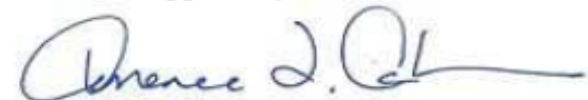

Signature applied by Donna L Stallings on 12/19/2008 10:17:31 AM CST

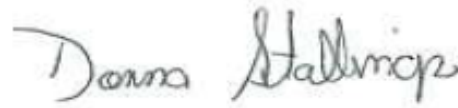

Terrence F. Ackerman, Ph.D.

Chairman

UTHSC IRB 
Appendix H. Approval Letter from the Pastor of St. John AME Church

St. John African

\section{Methodist Episcopal Church}

6070 St. John Chapel Road

Kushla, AL 36613

REV. IOHNNIE BRYANT, Pastor

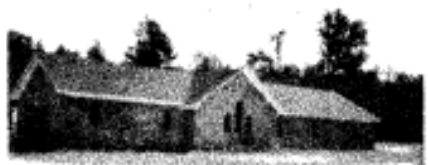

CHURCH: (205) 679-0588

1 November 2007

To Whom It May Concern,

Mrs. Bridget K. Robinson has been granted permission to inform the congregation of her study objectives and to collect information from those women who choose to participate. She also has permission to use the fellowship hall for study purposes.

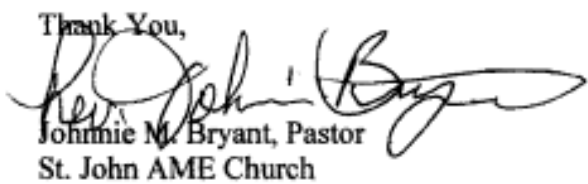




\section{VITA}

Bridget Katina Robinson was born Mobile, Alabama, on November 16, 1972. She was awarded a Bachelor of Science in Nursing and a Master of Science in Nursing Education from the University of South Alabama in Mobile, Alabama, in 2004 and 2005, respectively. She has over 5 years experience as a nurse. She has been employed in several areas of clinical nursing including: burn critical care, in-patient hemodialysis, and general medical-surgical.

She is eligible for certification as a Clinical Nurse Specialist in Adult Health with the AACN. She is the primary author on one unpublished manuscript and has presented at both national and international conferences.

Bridget K. Robinson received a Doctor of Philosophy in nursing degree from the University of Tennessee Health Science Center in May 2009. Her dissertation research focused on Physical Activity Behavior, Self-efficacy for Exercise, Religiosity, Body Image Perception, Body Mass Index, and Socioeconomic Factors among African American women. Presently she holds a fulltime faculty position at the University of South Alabama with primary teaching responsibilities in the undergraduate adult health nursing department. 